\title{
Algorithmic homeomorphism of 3-manifolds as a corollary of geometrization
}

\author{
Greg Kuperberg* \\ University of California, Davis
}

\begin{abstract}
In this paper we prove two results, one semi-historical and the other new. The semi-historical result, which goes back to Thurston and Riley, is that the geometrization theorem implies that there is an algorithm for the homeomorphism problem for closed, oriented, triangulated 3-manifolds. We give a self-contained proof, with several variations at each stage, that uses only the statement of the geometrization theorem, basic hyperbolic geometry, and old results from combinatorial topology and computer science. For this result, we do not rely on normal surface theory, methods from geometric group theory, nor methods used to prove geometrization.

The new result is that the homeomorphism problem is elementary recursive, i.e., that the computational complexity is bounded by a bounded tower of exponentials. This result relies on normal surface theory, Mostow rigidity, and bounds on the computational complexity of solving algebraic equations.
\end{abstract}

\section{INTRODUCTION}

In this paper, we will prove the following two theorems.

Theorem 1.1 (After Thurston [49]). Suppose that $M_{1}$ and $M_{2}$ are two finite, simplicial complexes that represent closed, oriented 3-manifolds. Then, as a corollary of the geometrization theorem, it is recursive to determine if there is an orientationpreserving homeomorphism $M_{1} \cong M_{2}$.

Theorem 1.2. The oriented homeomorphism problem for closed, oriented 3-manifolds is elementary recursive.

Theorem 1.1 implies that the geometrization theorem is a classification of closed, oriented 3-manifolds by the standard of computer science, where the term recursive used here means the same thing as decidable or computable. Geometrization intuitively presents itself as a classification of closed 3-manifolds, or at least a big step towards one. However, the question of what counts as a "classification" in mathematics is generally not rigorous, even though it is typically a debate over rigorous results. The computability interpretation is thus important because it is rigorous, even though it is not by any means the only important standard of classification. (For instance, the set of twin primes is recursive, but they remain unclassified in the sense that it is not even proven that there are infinitely many.) Note that Thurston himself [49, Sec. 3] seriously addressed the relation between geometrization and computability.

We argue that Theorem 1.1 should largely be credited to Riley and Thurston from the 1970s, even though they did not publish a complete proof. (See Section 1.1 for more details.) To support this interpretation, we will prove Theorem 1.1 directly using hyperbolic geometry; and using other background results concerning computability and triangulations of manifolds that seem standard and germane. The most important results of the latter type are the Tarski-Seidenberg theorem (Theorem 2.8) that real algebraic equations can be solved recursively, Kantorovich's theorem on convergence of Newton's

\footnotetext{
* greg@math.ucdavis.edu; Partly supported by NSF grants CCF-1319245 and CCF-1716990.
}

method (Theorem 5.8), and the stellar and bistellar move theorems of Alexander, Newman, and Pachner (Theorems 3.2 and 3.3). Despite this restriction on methods, we give more than one argument for each of several stages of the proof.

In the intervening years, Jaco-Tollefson, Manning, ScottShort, and others have published proofs of major parts of Theorem 1.1 [4, 18, 29, 43]. These approaches have various new ideas and implications, which is in keeping with Thurston's philosophy concerning the nature of progress in mathematics [50]. Even so, the status of Theorem 1.1 has remained unsettled. At one extreme, it has been interpreted as a folklore theorem and therefore standard knowledge, even if the proof is not elementary. At the other extreme, it has been interpreted as still an open problem. In the middle, one could argue that the published partial results piece together to make an entire proof. The problem with the middle position is that the total structure of an arbitrary closed, oriented 3-manifold is somewhat complicated; see Theorems 5.1, 5.2, and 5.3. So, one purpose of our proof of Theorem 1.1 is to give a complete proof in one paper, as requested by Aschenbrenner-FriedlWilton [4].

The intervening results also typically use either normal surface theory $[13,24]$ or geometric group theory $[8,11,46]$. While these methods certainly work, they arguably overshoot Theorem 1.1. Both theories are highly non-trivial in their own right, and they continued to be developed after the geometrization conjecture was stated. In particular the key results of Jaco-Tollefson [18] and Sela [46] came later. Sela's theorem applies to Gromov-hyperbolic groups, which are vastly more general than the Kleinian groups that arise in Theorem 1.1. Meanwhile Haken and Jaco-Tollefson prove sharper results than strictly necessary for their components of Theorem 1.1; namely, they establish algorithms with quantitative bounds on execution time. This brings us to Theorem 1.2.

In Theorem 1.2, an algorithm is elementary recursive if its execution time is bounded by a bounded tower of exponentials; for instance, time $O\left(2^{2^{n}}\right)$. (See Section 2.2.) In contrast with Theorem 1.1, the proof of Theorem 1.2 does use normal surface theory, as well as Mostow rigidity, and improved bounds on the computational complexity of solving algebraic equations [10]. The connected-sum and JSJ decomposition stages of Theorem 1.2 were partly known. For instance, using similar methods, Mijatović [31, 32] established an elementary 
recursive bounds on the number of Pachner moves needed to standardize either $S^{3}$ or a Seifert-fibered space with boundary.

The hyperbolic case of Theorem 1.2 is new. By contrast, Mijatović also established a primitive recursive bound on the number of Pachner moves needed to equate two hyperbolic, fiber-free, Haken 3-manifolds. However, primitive recursive is significantly weaker than elementary recursive; the Haken condition is also a significant restriction. Theorem 1.2 also has the advantage of combining a mixed set of methods to handle the full generality of closed, oriented 3-manifolds.

Remark. We leave the non-orientable versions of Theorems 1.1 and 1.2 for future work. This case includes new details such as 3-manifolds with essential, two-sided projective planes and Klein bottles. A more thorough result would also handle compact 3-manifolds with boundary.

\subsection{History and discussion}

As already mentioned, the geometrization conjecture has often been interpreted as a classification of closed 3manifolds, and computability is one candidate standard of what it means to classify mathematical objects. In Thurston's famous survey of his results in the AMS Bulletin [49, Sec. 3], he says:

Riley's work makes it clear that there is a rigorous, but not generally practical, algorithm for computing hyperbolic structures.

Thurston then sketches an algorithm which is similar Manning's construction [29] in some ways and to our arguments in other ways. This passage, and some other aspects of the Bulletin article, support the conclusion that Thurston anticipated not only the statement of Theorem 1.1, but also its proof. The author also discussed Theorem 1.1 in personal communication with Thurston in the late 1990s.

At first glance, an algorithm that can only find a hyperbolic structure on a 3-manifold is both less general and weaker than Theorem 1.1. It is less general because a 3-manifold may also have non-hyperbolic components; it is weaker because the homeomorphism problem for two hyperbolic manifolds $M_{1}$ and $M_{2}$ takes more work than just finding their hyperbolic structures. However, in the theorem that it is recursive to geometrize a 3-manifold (Theorem 5.4), the hyperbolic pieces (Lemma 5.7) are the hardest part. The geometric structure of the other pieces and the data to glue the pieces together are complicated to describe carefully, but the proof that this data is computable only requires moves on triangulations (Corollary 3.4), the principle that nested infinite loops can be combined into a single infinite loop (Proposition 2.5), and some facts about Seifert fibrations (Lemma 5.11 and Theorem 5.12).

In the second stage, the homeomorphism problem for hyperbolic 3-manifolds (Theorem 6.1) reduces to calculating isometries by Mostow rigidity, and a typical algorithm for this is similar to one for computing a hyperbolic structure. The homeomorphism problem for Seifert-fibered components and glued combinations of components (Section 6) requires little more than the ideas of Waldhausen [51] in his classification of graph manifolds.

Later in the Bulletin article [49, Sec. 6], Thurston gives a list of open problems and projects, including:

\section{Develop a computer program to calculate hy- perbolic structures on 3-manifolds.}

Jeff Weeks' SnapPea [53] (now SnapPy [6]), which was originally written in the $1980 \mathrm{~s}$, met this challenge. It is fast and reliable in practice, it can also compute the isometries between two hyperbolic 3-manifolds, and it has been supremely useful for a lot of research in 3-manifold topology. SnapPea also supports the belief that the homeomorphism problem follows from geometrization, given its spectacular record in practice. However, its specific algorithms are not rigorous. SnapPea uses ideal triangulations of cusped 3-manifolds, together with Dehn fillings to make spun triangulations of closed 3-manifolds; it is only conjectured that such a structure always exists. SnapPea also uses non-rigorous methods to find suitable triangulations. In particular it uses limitedprecision floating point arithmetic; it has no rigorous model of necessary precision as a function of geometric complexity. (Note that SnapPy has been extended to rigorously certify an answer, when SnapPea finds one.) In contrast to the SnapPea data structure, and other reasons that ideal and spun triangulations are important, we will use triangulations with semi-ideal and finite tetrahedra to prove Theorem 1.1 (see Section 5.2.5).

To start the rigorous discussion of computable classification and the homeomorphism problem, we can say that closed 3manifolds are classified if we can:

1. specify every closed 3-manifold by a finite data structure;

2. algorithmically generate a standard list of closed 3manifolds without repetition; and

3. given any 3-manifold $M$, algorithmically identify the standard manifold $M^{\prime}$ such that $M \cong M^{\prime}$.

For closed 3-manifolds, condition 1 is addressed by the fact that every 3-manifold has a unique smooth structure and a unique PL structure. As a result, we can describe a closed 3-manifold as a finite simplicial complex. Unlike in higher dimensions, it is easy to check whether a simplicial complex is a 3 -manifold (Section 3). Conditions 2 and 3 are equivalent to an algorithm to determine whether two closed 3-manifolds $M_{1}$ and $M_{2}$ are homeomorphic by the following simple argument. (Haken calls this argument the "cheapological trick" [52, Sec. 4].) In one direction, if both conditions 2 and 3 are satisfied, then Condition 3 immediately implies a homeomorphism algorithm. In the other direction, given a homeomorphism algorithm, we can make can lexicographically order all descriptions of all closed 3-manifolds according to condition 1 , and then list only those examples that are not homeomorphic to any earlier example. This satisfies condition 2. Then given a description of a closed 3-manifold $M$, we can search the list in order to find the standard $M^{\prime} \cong M$ to satisfy condition 3. (Haken calls this argument the "cheapological trick" 
[52, Sec. 4]. Arguably it is not cheap after all, since it is substantially similar to actual constructions of tables of knots and 3-manifolds.)

As mentioned, Manning [29] and Scott and Short [43] give partial results toward Theorem 1.1, but they use more recent tools. In particular, Manning uses Sela's algorithm [46] for the isomorphism problem for word-hyperbolic groups, while Scott and Short use the theory of biautomatic groups [8].

Both Short-Scott and Aschenbrenner-Friedl-Wilton [4, Sec. 2.1] mention a particular subtlety in approaches to Theorem 1.1 that are based on analyzing the fundamental group $\pi_{1}(M)$ or the fundamental groups of its components. Namely, $\pi_{1}(M)$ is insensitive to the orientation of $M$. Worse, if

$$
M \cong W_{1} \# W_{2} \# \ldots \# W_{n}
$$

is a decomposition of $M$ into prime summands, then the orientation of each summand $W_{k}$ can be chosen separately without changing $\pi_{1}(M)$. Or the summands can be lens spaces; two lens spaces can have the same fundamental group without even being unoriented homeomorphic. We surmount this subtlety by modelling all 3-manifolds and their components with triangulations that are decorated with orientations; see Section 5.2.

\section{ACKNOWLEDGMENTS}

The author would like to thank David Futer, Bus Jaco, Misha Kapovich, Jason Manning, Peter Scott, and Henry Wilton for very useful discussions. The author would also like to thank the anonymous referee for a careful reading that led to significant revisions.

\section{COMPUTABILITY}

We recommend Arora-Barak [3] and the Complexity Zoo [54] for modern introductions to models of computation and complexity classes.

\subsection{Recursive and recursively enumerable problems}

Let $A$ be a finite alphabet and let $A^{*}$ be the set of all finite words over that alphabet. A decision problem is a function

$$
d: A^{*} \rightarrow\{\text { yes, no }\}
$$

A function problem is likewise a function $f: A^{*} \rightarrow A^{*}$, which can be multivalued. The input space $A^{*}$ is equivalent to many other types of input by some suitable encoding: Finite sequences of strings, finite simplicial complexes, etc.

A decision problem or a function problem can be a promise problem, meaning that it is defined only on some subset of inputs $P \subseteq A^{*}$ which is called a promise. Whether two closed $n$-manifolds are PL homeomorphic is an example of a promise decision problem: The input consists of two simplicial complexes that are promised to be manifolds; then the yes/no decision is whether they are homeomorphic. (But see Proposition 3.1.)

A decision algorithm is a mathematical computer program, which can be modelled by a Turing machine (or some equivalent model of computation), that takes some input $x \in A^{*}$ and can do one of three things: (1) Terminate with the answer "yes", (2) terminate with the answer "no", or (3) continue in an infinite loop. Similarly, a function algorithm can terminate and report an output $y \in A^{*}$, or it can continue in an infinite loop. Given a multivalued function $f$, then a function algorithm is only required to calculate one of the values of $f(x)$ on input $x$.

A complexity class or computability class is some set of decision or function problems, typically defined by the existence of algorithms of some kind. For example, a decision problem $d$ or a function problem $f$ is recursive (or computable or decidable) if it is computed by an algorithm that always terminates. By definition, the complexity class $\mathrm{R}$ is the set of recursive decision problems. By abuse of notation, $\mathrm{R}$ can also denote the set of recursive, promise decision problems; or the set of recursive function problems, with or without a promise. The following proposition is elementary.

Proposition 2.1. If $d$ is a recursive promise problem, and if the promise itself is recursive, then $d$ is a recursive nonpromise problem if we let $d(x)=$ no when the promise is not satisfied.

The complexity class RE is the set of recursively enumerable decision problems. These are problems with an algorithm that terminates with "yes" when the answer is yes; but if the answer is "no", the algorithm might not terminate. The complexity class coRE is defined in the same way as RE, but with yes and no switched. We review the following standard propositions and theorems.

Proposition 2.2. A non-promise decision problem $d$ is in RE if and only if there is an algorithm that lists all solutions to $d(x)=$ yes without repetition.

Proposition 2.2 justifies the name "recursively enumerable" for the class RE. (Note that the solutions can be listed in order if and only if $d \in \mathrm{R}$.) The proof is left as an exercise. Also, in the spirit of Proposition 2.2, a decision problem $d$ can be identified with the set of solutions to $d(x)=$ yes; in this way we can call a set recursive, recursively enumerable, etc.

Proposition 2.3. $R=R E \cap$ coRE.

The proof of Proposition 2.3 is elementary but important: Given separate RE algorithms for both the "yes" and "no" answers, we can simply run them in parallel; one of them will finish. The proposition and its proof reveal the important point that a recursive algorithm might come with no bound whatsoever on its execution time.

Theorem 2.4 (Turing). The halting problem is in RE but not in $\mathrm{R}$. In particular, RE $\neq \mathrm{R}$. 
Informally, the halting problem is the question of whether a given algorithm with a given input terminates. Let $h$ be the halting decision problem, where the input $x$ in the value $h(x)$ is an encoding of an algorithm and its input (or, traditionally, an encoding of a Turing machine). It is easy to show that $h$ is $\mathrm{RE}$-complete in the following sense: Given a problem $d \in \mathrm{RE}$, there is a recursive function $f$ such that $d(x)=h(f(x))$ for any input $x$. Any other problem in RE with this same property can also be called RE-complete, or halting complete.

Proposition 2.5. Let $G$ be a graph structure on $A^{*}$. If the edge set of $G$ is recursively enumerable, then so is the set of pairs $(x, y)$, where $x$ and $y$ are vertices in the same connected component of $G$.

Proposition 2.5 is important for recursively enumerable infinite searches. The interpretation of the proposition, which is conveyed by the proof, is that nested infinite loops can be reorganized into a single infinite loop.

Proof. By Proposition 2.2, we can model a recursively enumerable set by an algorithm that lists its elements. The proposition states that the elements can be listed without repetition; but this is optional, since we can store all of the elements already listed and omit duplicates.

We use a recursive bijection $f$ between the natural numbers $\mathbb{N}$ and $\mathbb{N}^{*}$, the set of finite sequences of elements of $\mathbb{N}$. We can express any element of $\mathbb{N}^{*}$ uniquely in a finite alphabet that consists of the ten digits and the comma symbol. We can then list of all of these strings first by length, and then in lexicographic order for each fixed length. We can then let $f(n)$ be the $n$th listed string.

We can now convert the value $f(n)$ to a finite path $\left(x_{0}, \ldots, x_{k}\right)$ in the graph $G$, in such a way that every finite path is realized. If

$$
f(n)=\left(n_{0}, \ldots, n_{k}\right),
$$

then we let $x_{0}$ be the $n_{0}$ th string in $A^{*}$. For each $j>0$, we let $x_{j}$ be the $n_{j}$ th neighbor of $x_{j-1}$. In order to find the $n_{j}$ th neighbor of $x_{j-1}$, we list the elements of the edge set of $G$ until the edge $\left(x_{j-1}, x_{j}\right)$ arises as the $j$ th edge from $x_{j-1}$. There is the technicality that $x_{j-1}$ might not have an $n_{j}$ th neighbor if it only has finitely many neighbors. To avoid this problem, we intersperse trivial edges of the form $(x, x)$ infinitely many times, for every string $x \in A^{*}$, along with the non-trivial edges of $G$.

Since the algorithm finds every finite path in $G$, it finds every pair of vertices $x$ and $y$ in the same connected component. Thus, the set of such pairs is recursively enumerable.

\subsection{Elementary recursive problems}

As mentioned after Proposition 2.3, a recursive algorithm need not have any explicit upper bound on its execution time, beyond the tautological bound that running it is a way to calculate how long it runs. This motivates smaller complexity classes that are defined by explicit bounds. The most common notation for a bound on the execution time of an algorithm is asymptotic notation as a function of the input length $n=|x|$ to a decision problem $d(x)$. For example, we could ask for a polynomial-time algorithm, by definition one that runs in time $O\left(n^{k}\right)$ for some fixed $k$.

We have two reasons to consider a fairly generous bound in this paper. First, the recursive class $\mathrm{R}$ is unfathomably generous, so any explicit bound can be considered a major improvement. Second, the computational complexity of a problem or algorithm depends somewhat on the specific computational model, but certain relatively generous complexity bounds are substantially model-independent.

We consider a traditional Turing machine first. By (informal) definition, a Turing machine is a finite-state "head" with an infinite linear memory tape, and deterministic dynamical behavior. We say that an algorithm is elementary recursive if it runs in time

$$
O(\underbrace{2^{2^{\cdot 2^{n}}}}_{k})
$$

for some constant $k$. We call the corresponding complexity class ER. By abuse of terminology, we use ER to refer to both decision problems and function problems, and to numerical bounds. (Note that if $f(n)$ can be computed in ER, then a running time bound of $O(f(n))$ is itself a subclass of ER.)

Without reviewing rigorous definitions, we list some of the many variations in the computational model that do not affect the class ER in the following proposition. The proposition is not really needed in this paper except to motivate ER as an important complexity class. The only tacit dependence is that a random access machine is somewhat closer to both intuitive descriptions of algorithms and actual computers than a Turing machine with a linear tape is.

Proposition 2.6. Each of the following four computational models is the same as standard $\mathrm{ER}$.

1. A Turing machine with an n-dimensional tape, or a random access tape addressable by a separate address tape, with an elementary recursive bound on computation time.

2. A Turing machine restricted to an elementary recursive bound on computational space and unrestricted computation time.

3. A randomized Turing machine whose answers are probably correct, with an elementary recursive bound on computation time.

4. A quantum Turing machine that can compute in quantum superposition, with an elementary recursive bound on computation time.

Proof. Instead of a self-contained proof, we justify each case of the proposition with specific references to Arora-Barak [3].

1. This follows from Exercises 1.7 and 1.9 in Arora-Barak.

2. This follows from Theorem 4.2 in Arora-Barak. 
3. This reduces to case 4 by the proof of Corollary 10.11 in Arora-Barak.

4. This reduces to case 2 by the proof of Theorem 10.23 in Arora-Barak.

Remark. An elementary recursive bound is also a major improvement over another bound that is popular in logic and computer science: primitive recursive. An algorithm is primitive recursive if it runs in time $O(n[k] b)$ for some fixed $k$ and $b$, where the $k$ th operation $a[k] b$ is defined inductively as follows:

$$
\begin{gathered}
a[1] b=a+b \quad a[2] b=a b \quad a[3] b=a^{b} \\
a[k+1] b=\underbrace{a[k](a[k](\cdots(a[k] a) \cdots))}_{b} .
\end{gathered}
$$

For example, the operation $a[4] b$, which is called tetration, is defined as a tower of exponentials of height $b$. The primitive recursive complexity class is denoted PR. We can organize PR into a complexity hierarchy by defining $\mathrm{E}_{k}$ to be the set of functions computable in time $O(n[k+1] b)$ for some fixed $b$. Then $\mathrm{E}_{2}=\mathrm{P}, \mathrm{E}_{3}=\mathrm{ER}, \mathrm{E}_{4}$ consists of complexity bounds which are bounded towers of tetrations, etc.

\subsection{Computable numbers}

A computable real number $r \in \mathbb{R}$ is a real number with a computable sequence of bounding rational intervals. In other words, there is an algorithm that generates rational numbers $a_{n}, b_{n} \in \mathbb{Q}$ such that $x \in\left[a_{n}, b_{n}\right]$ and $b_{n}-a_{n} \rightarrow 0$. Many standard algorithms from numerical analysis, including field operations, integration of continuous functions, Newton's method, etc., have the property that if the input consists of computable numbers, then so does the output. One main limitation of computable real numbers is that inequality tests such as $a>b$ or $a \neq b$ are only recursively enumerable, not recursive. In other words, if $a \neq b$, then there is an algorithm to eventually confirm this fact and say which one is greater; but there is no terminating algorithm that always confirms that $a=b$.

We can avoid this shortcoming of the field of computable numbers by passing to a smaller subfield where equality is also recursive. In particular, we will use $\widehat{\mathbb{Q}}=\mathbb{R} \cap \overline{\mathbb{Q}}$, the real algebraic closure of the rational numbers $\mathbb{Q}$, which has this property.

Theorem 2.7. There is an encoding of the elements of $\widehat{\mathbb{Q}}$ such that field operations, order relations, and conversion to computable real numbers are all recursive.

One encoding of a real algebraic number $x$ that can be used to prove Theorem 2.7 is to describe it by a minimal polynomial together with an isolating interval $x \in[a, b]$ with rational endpoints to distinguish $x$ from its Galois conjugates. Note that the isolating interval may be made arbitrarily small since algebraic numbers are computable, for instance by Newton's method. Note also that a computable encoding of elements of $\hat{\mathbb{Q}}$ yields a computable encoding of elements of $\overline{\mathbb{Q}} \subseteq \mathbb{C}$ the field of all algebraic numbers.
Remark. The field of real algebraic numbers together with reliable equality testing is implemented in Sage [41].

Theorem 2.8 (Tarski-Seidenberg [44, 47]). It is recursive to determine whether there is a solution to a finite list of polynomial equalities and inequalities with coefficients in $\widehat{\mathbb{Q}}$ in finitely many variables; or to find a solution.

Actually, Tarski and Seidenberg proved the stronger result that it is recursive to decide any assertion over $\mathbb{R}$ expressed with polynomial relations and first-order quantifiers.

\section{TRIANGULATIONS OF MANIFOLDS AND MOVES}

In this section, we will analyze the form of the input to Theorem 1.1. We will show that given simplicial complexes as input to the homeomorphism problem, we can first confirm that they are 3-manifolds. (It is also easy to confirm that input strings actually represent simplicial complexes, in some convenient data type.) Thus Proposition 2.1 applies: we can view the homeomorphism problem as a non-promise problem. Actually, Proposition 3.1 below is overkill for this purpose, since it is much harder in dimension $n=4$ than in dimension $n=3$.

We then discuss moves between triangulations of a manifold, mainly to establish Corollary 3.4. In light of Proposition 2.3, Corollary 3.4 is an easy half of Theorem 1.1, one that holds in any dimension $n$.

Proposition 3.1. If $\Theta$ is a finite simplicial complex of dimension $n \leq 4$, then it is recursive to determine whether it is a closed PL n-manifold, and whether or not it is orientable.

Proof. The proof is partly by induction on dimension $n$. The result is trivial if $n=0$, where we need only check that $\Theta$ is a single point. Otherwise, we first check that $\Theta$ is connected, and we must check that the link $\Lambda$ of every vertex is both a closed $(n-1)$-manifold and a PL $n$-sphere. The former condition is the inductive step. The latter condition requires an algorithm to recognize an $(n-1)$-sphere. If $\Lambda$ is a closed 1manifold, then it is immediately a 1 -sphere, i.e., a circle. If $\Lambda$ is a closed 2-manifold, then we can compute its Euler characteristic. If $\Lambda$ is a closed 3-manifold, then Theorem 1.1 implies that it is recursive to determine if $\Lambda$ is a 3 -sphere, although this result was obtained without geometrization by Rubinstein and Thompson (Theorem 8.7) [40, 48].

We can check that $\Theta$ is orientable (and orient it) algorithmically by computing its simplicial homology.

The stellar and bistellar subdivision theorems establish that every two triangulations of a compact $n$-manifold, in particular a compact 3-manifold, are connected by a finite sequence of explicit moves. See Lickorish [28] for a modern treatment and a historical review.

Theorem 3.2 (Alexander-Newman). If two finite simplicial complexes $\Theta_{1}$ and $\Theta_{2}$ are PL equivalent, then they are connected by a sequence of stellar subdivision moves and their inverses. 
Briefly, a stellar move in a simplicial complex $\Theta$ consists of replacing the star st $(\Delta)$ of some simplex $\Delta$ in $\Theta$ with a cone over the subcomplex of simplices in $\operatorname{st}(\Delta)$ that do not contain $\Delta$. Equivalently, the apex $v$ of this cone is placed in the interior of $\Delta$, and all simplices that contain $\Delta$ are subdivided to support the new vertex $v$.

Theorem 3.3 (Pachner). If $\Theta_{1}$ and $\Theta_{2}$ are two triangulations of a compact, PL manifold $M$, then they are connected by bistellar moves.

A bistellar move of a triangulation of an $n$-manifold $M$ consists of a stellation followed an inverse stellation at the same vertex. Equivalently, two triangulations of $M$ differ by a bistellar move when there is a minimal cobordism between them consisting of a single $(n+1)$-simplex. In particular, a shellable triangulation of $M \times I$ yields a sequence of bistellar moves.

Lickorish points out that Newman conjectured and partially proved Theorem 3.3 in an earlier paper, before he and Alexander separately proved Theorem 3.2. Bistellar moves are also called Pachner moves, although arguably they should be called Newman-Pachner moves.

Theorem 3.3 also holds for ideal or semi-ideal triangulations of a compact 3-manifold with torus boundary components. (In other words, it holds for a 3-dimensional pseudomanifold with singular points with torus links, which are the ideal vertices.)

Theorems 3.2 and 3.3 each have the following corollary.

Corollary 3.4. The PL homeomorphism problem for compact PL n-manifolds is in RE.

Remark. There is a proof of Corollary 3.4 that works directly from the definition of PL equivalence without using Theorem 3.2 or 3.3 , nor even Proposition 2.5. For each $n$, choose a linear embedding of an $n$-simplex $\Delta^{n} \subseteq \mathbb{R}^{n}$. Then in general a geometric refinement is a simplicial complex $\Theta$ with a homeomorphism onto $\Delta$ which is affine-linear on each simplex of $\Theta$. Likewise a refinement of a simplicial complex $\Theta_{1}$ is another simplicial complex $\Theta_{2}$ with a homeomorphism $f: \Theta_{2} \rightarrow \Theta_{1}$, such that $f$ yields a geometric refinement of each simplex of $\Theta_{1}$. By definition, two complexes $\Theta_{1}$ and $\Theta_{2}$ are PL equivalent if they share a refinement $\Theta_{3}$. Now, we can let each $\Delta^{n}$ have rational vertices (i.e., vertices in $\mathbb{Q}^{n}$ ), and after that we can perturb any geometric refinement so that its vertices are all rational. The set of rational mutual refinements of two finite complexes $\Theta_{1}$ and $\Theta_{2}$ is recursive by direct verification. (In other words, given a simplicial complex $\Theta_{3}$, and given rational target positions for its vertices in both $\Theta_{1}$ and $\Theta_{2}$, we can algorithmically check whether this data yields a mutual refinement.) Therefore the question of whether there exists a mutual refinement is directly recursively enumerable.

Proposition 3.5. If $\Theta_{1}$ is a finite simplicial complex with $n_{1}$ simplices (of arbitrary dimension) and $n_{2} \geq n_{1}$, then it is recursive to produce a complete list of geometric subdivisions $\Theta_{2}$ of $\Theta_{1}$ with $n_{2}$ simplices.

Proof. There are only finitely many simplicial complexes $\Theta_{2}$ with $n_{2}$ simplices, and they can be generated recursively. For each candidate for $\Theta_{2}$, there are only finitely many combinatorial choices for a function from the simplices of $\Theta_{2}$ to the simplices of $\Theta_{1}$. For each such choice, we can first check that the simplices of $\Theta_{2}$ that land in a $k$-simplex $\Delta \in \Theta_{1}$ support a simplicial cycle that represents the fundamental class in $H^{k}(\Delta, \partial \Delta)$. We solve for each such cycle for all $\Delta$ (where each must be unique if $\Theta_{2}$ indeed subdivides $\Theta_{1}$ ). Then the constraint that each simplex of $\Theta_{2}$ must be positively oriented in $\Theta_{1}$ yields we obtain algebraic inequalities for the positions of all vertices. We can then apply Theorem 2.8 to see if there is a solution for those positions.

We conclude this section with the following theorem which combines results of P.S. Novikov, Boone, Adian, Rabin, Markov, and S.P. Novikov [39].

Theorem 3.6 (NBARMN). The isomorphism problem for finitely presented groups, the PL homeomorphism problem for 4-manifolds, and the recognition of $S^{n}$ among PL n-manifolds for each $n \geq 5$ are all halting-complete.

It is not known whether either topological or PL recognition of $S^{4}$ is recursive.

Remark. The homeomorphism problem for PL $n$-manifolds in Theorem 3.6, or even recognition of $S^{n}$, needs to be handled with some care, for several reasons. First, because recognizing whether the input is a PL $n$-manifold is (by Theorem 3.6!) an uncomputable promise when $n \geq 6$. Second, because there are closed manifolds that are homeomorphic but not PL homeomorphic [23]. Third, because there are simplicial complexes that are not PL $n$-manifolds at all, but that are homeomorphic to $S^{n}$, for each $n \geq 5$ [7]. The proof of Theorem 3.6 dispenses with all of these concerns as follows. Given an input $x$ to the halting problem $h(x)$ and an integer $n \geq 4$, there is an algorithm that constructs an $n$-manifold $M(x)$ such that:

1. $M(x)$ is manifestly a closed PL manifold.

2. $M(x)$ is PL homeomorphic to $S^{n}$ when $n \geq 5$, or to a connected sum of copies of $S^{2} \times S^{2}$ when $n=4$, if and only if $M(x)$ is simply connected.

3. $M(x)$ is simply connected if and only if $h(x)=$ yes.

Remark. By contrast with Theorem 3.6, the PL homeomorphism problem for simply connected $n$-manifolds with $n \geq 5$ is recursive [36].

\section{SOME NOTATION}

We summarize some notation for specific topological spaces, beyond the most standard notation that $S^{n}$ is an $n$ sphere, $D^{n}$ is an $n$-disk, $P^{n}$ is real projective $n$-space, and $I=D^{1}$ is an interval.

We let $X \ltimes Y$ denote a fiber bundle with base $X$ and fiber $Y$. Although the notation $X \tilde{\times} Y$ is reasonably standard for a twisted bundle, we prefer to write $X \ltimes Y$, for two reasons. First, because the notation specifies which side is the fiber; we can write $X \ltimes Y \cong Y \rtimes X$. Second, because a fiber bundle is analogous to a semidirect product in group theory. 
We review Seifert's description of oriented Seifert-fibered spaces [45]. If $F$ is a compact surface which may or may not be orientable, then there is a unique, canonically oriented $I$ bundle $F \ltimes I$. If $F$ is orientable, then this $I$-bundle is simply $F \times I$; in this case we assume an orientation for the base $F$ and the fiber $I$. We consider the double $F \ltimes S^{1}$ of $F \ltimes I$, which again when $F$ is orientable is just $F \times S^{1}$.

If $p_{1}, p_{2}, \ldots, p_{n}$ are points of $F$, then we can apply a Dehn surgery with slope $b_{k} / a_{k}$ to a solid torus neighborhood of the fiber over $p_{k}$ in $F \ltimes S^{1}$, where $a_{k}$ is a positive integer and $b_{k}$ is a relatively prime integer of either sign. The resulting oriented 3-manifold $N$ is thus construction from its Seifert data, namely the multiset

$$
\left\{F,\left(a_{1}, b_{1}\right),\left(a_{2}, b_{2}\right), \ldots,\left(a_{n}, b_{n}\right)\right\} .
$$

In general we interpret $F$ as an orbifold. If $a_{k} \geq 2$, then we interpret $p_{k} \in F$ as an orbifold point of order $a_{k}$, and the circle over it is an exceptional fiber. By Seifert's classification, the integers $a_{k}$ with $a_{k} \geq 2$ together with the residues $b_{k} \in \mathbb{Z} / a_{k}$ are all topological invariants of the fibration of $N$. If $F$ and therefore $N$ has boundary, then this is a complete set of invariants. If $N$ is closed, then the Euler number

$$
e(N)=b+\sum_{k} \frac{b_{k}}{a_{k}}
$$

is the only additional necessary invariant. Thus there is a canonicalized version of the Seifert data in the form

$$
\left\{F, b,\left(a_{1}, b_{1}\right),\left(a_{2}, b_{2}\right), \ldots,\left(a_{n}, b_{n}\right)\right\},
$$

where $b$ represents $(1, b)$ and otherwise $a_{k} \geq 2$ and $1 \leq b_{k}<$ $a_{k}$. If $F$ is non-compact, then $b$ is irrelevant and we omit it in the canonical form.

With the notation of fiber bundles and Seifert-fibered spaces, we name these specific manifolds:

1. We use $S^{1} \times S^{1}$ to denote the standard 2-torus, and $T$ to denote an arbitrary 2-torus, i.e., $T \cong S^{1} \times S^{1}$.

2. $K^{2}=S^{1} \ltimes S^{1}$ is the 2-dimensional Klein bottle.

3. $L(m, n)$ is the lens space defined by the Seifert data $\left\{S^{2}, 0,(n, m)\right\}$.

4. $R(m, n)$ denotes the prism space defined by the Seifert data $\left\{P^{2}, 0,(m, n)\right\}$.

\section{GEOMETRIZATION IS RECURSIVE}

The goal of this section is to prove Theorem 5.4, which says that the geometric decomposition of a 3 -manifold $M$ is computable.

\subsection{Statement of geometrization}

We begin with three results that, together, are one formulation of the geometrization theorem for closed, oriented 3manifolds.
Theorem 5.1 (Kneser-Milnor [24, 34]). Every closed, oriented 3-manifold (other than $S^{3}$ ) is a connected sum of prime, closed, oriented 3-manifolds (none of which are $S^{3}$ ). The summands are unique up to oriented homeomorphism.

We will adopt the convenience that a 3 -sphere $S^{3}$ counts as a prime 3-manifold, notwithstanding that Theorem 5.1 would be easier to state if $S^{3}$ were instead interpreted as the "unit" in the terminology of unique factorization.

Theorem 5.2 (Jaco-Shalen-Johansson [19, 20]). A closed, oriented, prime 3-manifold has a minimal collection of incompressible tori, unique up to isotopy and possibly empty, with the property that the complementary regions are either Seifert-fibered or atoroidal.

The decomposition in Theorem 5.2 is called the JSJ decomposition. We can call the tori JSJ tori, and the complementary regions JSJ components. We will use $M$ to denote a general closed, oriented 3-manifold; then $W$ to denote a prime summand of $M$; then $N$ to denote a JSJ component of $W$.

Theorem 5.3 (Thurston-Hamilton-Perelman). Suppose that $N$ is an oriented, prime, atoroidal 3-manifold which is either closed or has torus boundary components. Then $N$ is either Seifert-fibered, or it is closed and has a unique hyperbolic structure, or its interior $N^{*}$ has a unique, complete hyperbolic structure with torus cusps.

As everyone knows, Theorem 5.3 was conjectured and partly proven by Thurston [49], then fully proven by Perelman using the Ricci flow program of Hamilton [35]. (Note that Theorem 5.3 implicitly includes the Poincaré conjecture in the Seifert-fibered case.)

Remark. Mixing the JSJ decomposition with hyperbolization is a less pure approach than Thurston's decomposition into geometric components, but we find it convenient for Theorem 1.1. We could recognize spherical and Euclidean components with the same methods as hyperbolic components (Lemma 5.7), while several of the other Thurston geometries induce canonical Seifert fibrations. In fact, every Seifertfibered 3-manifold or component is geometric. Conversely, every geometric 3-manifold or component is hyperbolic unless it is Seifert-fibered or a Sol manifold.

\subsection{Statement of computational geometrization}

Theorem 5.4. If $M$ is a triangulated 3-manifold, then it is recursive to compute a decorated triangulation which is adapted to its geometric decomposition.

Before proving Theorem 5.4, we need to state it more precisely. When $\Theta$ is a decorated, adapted triangulation of $M$ it means that:

1. $M$ has a distinguished (but possibly empty) collection of disjoint, separating 2-spheres, each triangulated with 4 triangles in $\Theta$, that separates it into prime summands $\{W\}$. Each $W$ is closed; it inherits its triangulation from $\Theta$ and its holes are plugged with fresh tetrahedra. 
2. The triangulation of each $W$ supports a distinguished (but possibly empty) collection of disjoint thickened tori $T \times I$ and restricts to a shelled triangulation of each one. These thickened tori separate $W$ into JSJ components $\{N\}$.

3. The tetrahedra at all stages are consistently oriented, to express an orientation of each summand $W$ and each JSJ component $N$ that is consistent with the orientation of $M$.

4. If $N$ is Seifert-fibered with base $F$, then we make a triangulation which is adapted to Seifert's description of $N$ by Dehn surgery on $F \times S^{1}$ when $F$ is orientable, or Dehn surgery on a twisted bundle $F \ltimes S^{1}$ when $F$ is nonorientable. This includes the case where $N=W=M$ is a 3-sphere.

5. If $N$ is hyperbolic, then it is marked as the barycentric subdivision of a regular, adapted cellulation $\Lambda$. $\Lambda$ comes from a geometric triangulation $\Lambda^{*}$ of $N^{*}$ in which each tetrahedron has at most one ideal vertex. If $\Delta \in \Lambda^{*}$ has an ideal vertex, then it is truncated to a triangular prism in $\Lambda$; if not, then it is kept in $\Lambda$. Each tetrahedron in $\Lambda^{*}$ is also decorated with its dihedral angles.

We proceed to explain each stage of the definition.

\subsubsection{The prime decomposition}

Note that we take a triangulation to be a simplicial complex structure rather than a generalized triangulation. In geometric topology, for instance in the SnapPea census, a generalized triangulation is sometimes defined to be a CW-complex whose cells are simplices and whose attaching maps take simplices to simplices. In particular, a simplex in a generalized triangulation need not have distinct vertices and two simplices may have the same vertices. We can form a connected sum of two triangulated 3-manifolds by removing a single tetrahedron from each one and gluing the sphere boundaries.

\subsubsection{Shelled triangulations}

If $X$ is a closed $n$-manifold with two triangulations $\Theta_{0}$ and $\Theta_{1}$, then a shelled triangulation of $X \times I$ is a simplicial complex $\Theta_{0,1}$ whose $(n+1)$-simplices are numbered. Taken in order, the $(n+1)$-simplices connect the triangulation $\Theta_{0}$ of $X \times\{0\}$ to the triangulation $\Theta_{1}$ of $X \times\{1\}$ via a sequence of bistellar moves. Note that this combinatorial restriction on $\Theta_{0,1}$ implies $\Theta_{0,1}$ is PL homeomorphic to $X \times I$. In other words, if we build $\Theta_{0,1}$ from a sequence of bistellar moves, and if $X \times\{0\}$ and $X \times\{1\}$ are disjoint in the result, then $\Theta_{0,1}$ is a triangulation of $X \times I$.

\subsubsection{Orientations}

To be precise, we can decorate each tetrahedron by ordering its vertices, where two orderings are equivalent if they differ by an even permutation.

\subsubsection{Seifert-fibered components}

We begin with preliminaries on cellulations and barycentric subdivisions that we will also need in Section 5.2.5.

A cellulation of a topological space $X$ is a CW complex $\Lambda$ with a homeomorphism to $X$. The complex $\Lambda$ is regular if $\Lambda$ is locally finite; and if the attaching map of each $k$-cell is an embedding in the $(k-1)$-skeleton, so that each closed $k$-cell of $\Lambda$ is embedded in $X$.

We will use the following standard proposition to model regular CW complexes using triangulations.

Proposition 5.5 ([26, Sec. 10.3.5]). Every regular CW complex $\Lambda$ has a barycentric subdivision $\Theta$ which is a simplicial complex, and the spaces of $\Theta$ and $\Lambda$ are homeomorphic.

See Figure 1 for an example.
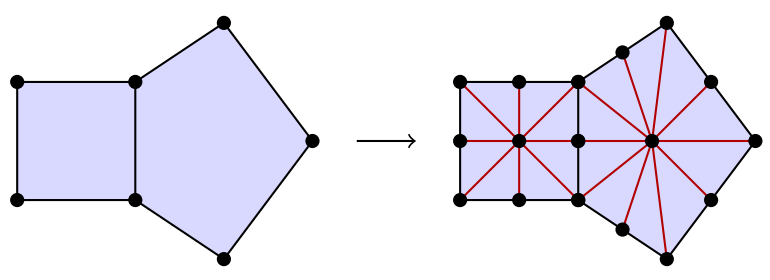

Figure 1. A barycentric subdivision of part of a cellulation of a surface.

If $N$ is a Seifert-fibered component, then as described in Section 4, it has a base orbifold $F$ with one circle for each boundary torus of $N$. The fibration has canonical Seifert data

$$
\left\{F, b,\left(a_{1}, b_{1}\right),\left(a_{2}, b_{2}\right), \ldots,\left(a_{n}, b_{n}\right)\right\},
$$

with $b$ omitted when $F$ or $N$ has boundary. The data indicates surgery with slope $b_{k} / a_{k}$ at the fiber over some $p_{k} \in F$ and (if it exists) surgery with slope $b$ at $p_{0} \in F$.

We choose a triangulation $\Theta_{F}$ of $F$ such that each $p_{k}$ (including $p_{0}$, if it exists) lies in the interior of a triangle, and such that all of these triangles are disjoint. We can lift $\Theta_{F}$ to a cellulation $\Lambda$ such that the solid torus $\Delta \times S^{1}$ over each triangle $\Delta$ in $\Theta_{F}$ is tiled by two vertical triangular prisms. We take the barycentric subdivision of $\Lambda_{F}$ to obtain a triangulation of $F \times I$ or $F \ltimes I$. If a triangle $\Delta \in \Lambda$ contains some $p_{k}$, we remove the solid torus $\Delta \times S^{1}$ (which is now triangulated with 72 tetrahedra) and glue it back using Dehn surgery. The gluing involves a homeomorphism of the boundary $\partial\left(\Delta \times S^{1}\right)$, which we implement with a shelled triangulation of a thickened torus, as in Section 5.2.2. The result is a triangulation of $N$, which we decorate with information about how it was constructed, so that the canonical Seifert data is part of the decoration. 


\subsubsection{Hyperbolic components}

If the component $N$ is hyperbolic, then we choose a geometric triangulation $\Theta^{*}$ of $N^{*}$, meaning one whose tetrahedra lift to geometric tetrahedra in the universal cover $\mathbb{H}^{3}$. More precisely, if $\hat{N}$ is the compactification of $N$ given by collapsing each torus boundary component to a point, we assume a continuous map

$$
f: \Theta^{*} \rightarrow \hat{N}
$$

such that the image $f(\Delta)$ of each combinatorial tetrahedron $\Delta$ lifts to a geometric tetrahedron in the standard compactification $\overline{\mathbb{H}^{3}}$ of hyperbolic space. If none of the vertices of $f(\Delta)$ are at infinity, then $f(\Delta)$ is finite; if they are all at infinity, then $f(\Delta)$ is ideal; and if some are at infinity, then $f(\Delta)$ is semiideal. We will assume that all of the simplices of our $\Theta^{*}$ are either finite or semi-ideal with one ideal vertex. If $N=N^{*}$ is closed, then all tetrahedra in $\Theta^{*}$ must be finite; if $N$ has boundary components and thus $N^{*}$ has cusps, then some of the tetrahedra must be semi-ideal.

Before proceeding further, we contrast this with some other models that have also been studied as geometric triangulations. In some treatments $f$ is not a homeomorphism but only a homotopy equivalence. In the case we can still ask for the restriction of $f$ to each tetrahedron $\Delta$ to be affine-linear in the Klein model of $\overline{\mathbb{H}^{3}}$. However, $f(\Delta)$ may be degenerate, meaning that it has zero volume, or it may be flipped over, meaning that it has negative signed volume relative to the orientation of $\Theta^{*}$ and the standard orientation of $\mathbb{H}^{3}$. In another variation, which is often used when $N$ is closed, the inverse map $g: \hat{N} \rightarrow \Theta^{*}$ is defined, and a finite set of closed geodesic curves in $\hat{N}$ collapse to ideal points; but the inverse image of any open tetrahedron in $\Theta^{*}$ is still a geometric tetrahedron in $\hat{N}$. Such a structure is a spun triangulation, because a geodesic circle $C \subseteq N$ is approached by cusps of ideal tetrahedra that wind helically around it. In particular SnapPea uses spun triangulations.

Ideal geometric triangulations are especially desirable in computational hyperbolic geometry because they are rigid and algebraically the simplest. However, it is only a conjecture that every suitable hyperbolic manifold has an ideal, possibly spun geometric triangulation. Such a structure does always exist with degenerate or flipped-over tetrahedra, but these are less desirable. We will use finite and semi-ideal tetrahedra in order to avoid this impasse. The following proposition is then standard:

Proposition 5.6. If $N^{*}$ is a complete hyperbolic manifold which is either cusped or closed, then it has a geometric triangulation with finite and semi-ideal tetrahedra (none of which are spun, degenerate, or flipped over). Also, every semi-ideal tetrahedron has only one ideal vertex.

Proof. We can choose a point $p \in N^{*}$ and consider the Voronoi tiling of its orbit in $\mathbb{H}^{3}$. Each Voronoi cell is a fundamental domain and yields a cellulation $\Lambda_{1}$ of $\hat{N}$. $\Lambda_{1}$ is not in general regular, but it has a barycentric subdivision $\Lambda_{2}$ which is regular. Moreover, each simplex of $\Lambda_{2}$ has at most one vertex of
$V$ and thus at most one ideal vertex. We can let $\Theta=\Lambda_{3}$ be a second barycentric subdivision, which is then a simplicial complex and still has the property that each tetrahedron has at most one ideal vertex.

Given a semi-ideal triangulation of $N^{*}$, we can truncate the cusps so that each semi-ideal tetrahedron becomes a triangular prism, as in Figure 2. A barycentric subdivision of this cellulation is then the desired adapted triangulation.

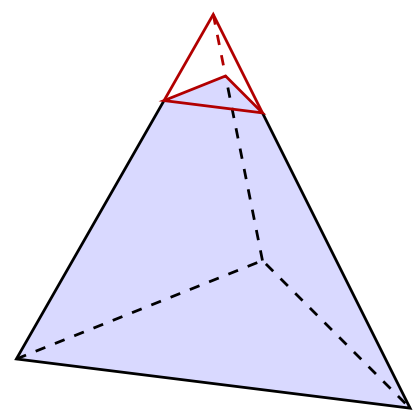

Figure 2. A tetrahedron truncated at one vertex.

\subsection{Proof of Theorem 5.4}

Lemma 5.7. It is recursive to find a geometric triangulation of a hyperbolic 3-manifold $N$ which is either closed or has torus boundary components, using either of two descriptions of each dihedral angle $0<\alpha<\pi$ of each tetrahedron:

1. Each imaginary exponential $\exp (i \alpha)$ is specified as an element of $\overline{\mathbb{Q}}$.

\section{Each angle $\alpha$ is given as a computable real number.}

Hence, it is in RE to determine if $N$ is hyperbolic.

Although the second case of Lemma 5.7 immediately follows from the first one, we will give a separate proof of each case. Moreover, even the weaker second case of Lemma 5.7 is sufficient to prove Theorem 1.1.

Remark. Manning [29, Thm. 5.2] also proves Lemma 5.7, but as a corollary of a harder result. His results show (without geometrization) that it is recursive to decide whether $N$ is hyperbolic, when there is an algorithm for the word problem for $\pi_{1}(N)$. He also uses a single polyhedral fundamental domain to describe the geometry of $N$. Although this differs from a hyperbolic triangulation, which is what we use, the two models are somewhat interchangeable for our purposes.

Proof of case 1 of Lemma 5.7. Suppose that $\Theta^{*}$ is a geometric triangulation of $N^{*}$. We can model each tetrahedron $\Delta \in \Theta^{*}$ (non-uniquely) by choosing four vertices in the Poincaré upper half-space model, including one on the boundary if $\Delta$ is semi-ideal. (Note that the ideal vertices of $\Theta^{*}$ are marked in advance.) There is an algebraic formula for each finite edge 
length $\ell$ and each dihedral angle $\alpha$ of $\Delta$, if these are represented by their exponential values $\exp (\ell)$ and $\exp (i \alpha)$. The main matching condition for $\Theta^{*}$ to be geometric is that if two tetrahedra share a finite edge, then the edge lengths agree; and the total dihedral angle around each edge equals $2 \pi$. The first condition is immediately an algebraic condition, although note that if an edge is semi-ideal, then it has infinite length and its length equation is vacuous.

The second condition is almost an algebraic condition since the product of the exponentiated angles must be 1; this shows that the total angle is a multiple of $2 \pi$, although not which one. However, this can be remedied with additional algebraic inequalities, recalling that we are allowed real algebraic equations for the real and imaginary parts $\cos (\alpha)$ and $\sin (\alpha)$ of each complex variable $\exp (i \alpha)$. Suppose that every edge of $\Theta^{*}$ has at most $n$ incident tetrahedra. Then we can make a finite covering of the unit circle $S^{1} \subseteq \mathbb{C}$ by rational rectangles such that each one has an angular extent of less than $2 \pi / n$. We can then loop over choices for which rectangle contains each exponentiated angle $\exp (i \alpha)$. If each angle is confined to such a rectangle, we can know whether the sum of the angles around an edge is specifically $2 \pi$ and not some other multiple of $2 \pi$.

After forming algebraic equations for all of the geometric data, the equations have a solution in terms of real algebraic numbers when they have a solution at all. For any fixed triangulation $\Theta$, we can thus use Theorem 2.7 (not Theorem 2.8; see the remark after the proof) to search for a solution and eventually find it, if it exists. We must also search over triangulations using Theorem 3.2 or Theorem 3.3. Since the result is a nested infinite search (over triangulations and then candidate geometric structures), we can apply Proposition 2.5.

Remark. Although an infinite search for a solution to algebraic gluing equations is preposterous in practice, it is good enough for an algorithm in RE. Alternatively, for each triangulation, we can apply the more difficult Theorem 2.8 to determine in $\mathrm{R}$ if there is a solution.

Remark. If we allowed geometric triangulations with fully ideal edges, then it would not be enough for the sum of the angles around such an edge $e$ to be $2 \pi$. Since $e$ goes to itself under hyperbolic translation as well as rotation, gluing together the tetrahedra that contain $e$ could create a non-trivial translational holonomy. The two conditions together, that the total angle is $2 \pi$ and the translational holonomy vanishes, are known as a Neumann-Zagier gluing relation [38].

Remark. Instead of calculating lengths and angles using positions of vertices in hyperbolic geometry, we can also relate them directly using formulas from hyperbolic and spherical trigonometry.

The separate proof of the second case of Lemma 5.7 works directly with computable numbers, in effect using numerical analysis to calculate better and better approximate solutions. In this approach, we need a criterion to know that an approximate solution is close to an exact one. Given a smooth multivariate equation $f(x)=0$, the Newton-Kantorovich theorem [21] establishes a sufficient criterion for Newton's method to converge from an approximate solution $x_{0}$ to an exact solution $x_{\infty}$. Neuberger [37] points out that an ODE analogue of Newton's method, which is called the continuous Newton's method, simplifies the Newton-Kantorovich result.

Theorem 5.8 (Newton-Kantorovich-Neuberger [37, Thm. 2]). Let $B_{\varepsilon}\left(x_{0}\right) \subset \mathbb{R}^{n}$ be the open ball of radius $\varepsilon>0$ around $x_{0} \in$ $\mathbb{R}^{n}$, and let

$$
f: B_{\varepsilon}\left(x_{0}\right) \rightarrow \mathbb{R}^{n}
$$

be a $C^{2}$-smooth function with non-singular matrix derivative Df. Suppose that

$$
\left\|(D f(x))^{-1} f\left(x_{0}\right)\right\|<\varepsilon
$$

for all $x \in B_{\varepsilon}\left(x_{0}\right)$, where $\|\cdot\|$ is the Euclidean norm on $\mathbb{R}^{n}$. Then there is a unique $x_{\infty} \in B_{\varepsilon}\left(x_{0}\right)$ such that $f\left(x_{\infty}\right)=0$. Also, given a solution $x_{\infty}$ such that $D f\left(x_{\infty}\right)$ is non-singular, equation (1) eventually holds as $x_{0} \rightarrow x_{\infty}$, moreover with $\varepsilon \rightarrow 0$.

Although we will not reprove Theorem 5.8, we can discuss where the theorem and its proof come from. Newton's method to find a root of a univariate function $f:(a, b) \rightarrow \mathbb{R}$ begins at an approximate root $x_{0} \in(a, b)$ and applies the iteration

$$
x_{n+1}=x_{n}-\frac{f\left(x_{n}\right)}{f^{\prime}\left(x_{n}\right)},
$$

which in favorable cases converges to a solution $x_{\infty}$ of the equation $f(x)=0$. If $f$ is multivariate as in Theorem 5.8, then this has the well-known matrix generalization

$$
x_{n+1}=x_{n}-\left(D f\left(x_{n}\right)\right)^{-1} f(x) .
$$

Finally in the continuous version, we let $x(0)=x_{0}$ and define the ODE

$$
x^{\prime}(t)=-(D f(x(t)))^{-1} f(x) .
$$

Then in favorable cases the limit

$$
x_{\infty}=\lim _{t \rightarrow \infty} x(t)
$$

is again a solution to $f(x)=0$.

Remark. Although Neuberger's paper on the continuous Newton's method is more recent than Thurston's work, Kantorovich's earlier, more complicated formula also suffices for Lemma 5.7 and Theorem 1.1.

If the equation $f(x)=0$ has a non-singular Jacobian $D f$ in a neighborhood of a solution, as in Theorem 5.8, then the system of equations is also called transverse or first-order rigid. We will need a generalization of this concept. Given a smooth function

$$
U \subseteq \mathbb{R}^{n} \quad f: U \rightarrow \mathbb{R}^{m}
$$

where $n$ and $m$ need not be equal, if $D f$ has constant rank $k r$, then the image $f(U)$ is a manifold and $f$ is a submersion onto its image. In this case the equation $f(x)=0$ is first-order rigid except for the directions parallel to the manifold $f^{-1}(0)$. 
By the implicit function theorem, we can discard some set of $n-r k$ coordinates in the domain and project to some set of $k$ coordinates in the target to achieve unconditional first-order rigidity that satisfies the hypotheses of Theorem 5.8.

To establish first-order rigidity in our case, we will need a corollary of the Calabi-Weil rigidity theorem.

Theorem 5.9 (Calabi-Weil [22, Sec. 8.10]). If $N$ is a closed, hyperbolic 3-manifold, then the induced representation of its fundamental group,

$$
\rho: \pi_{1}(N) \rightarrow \operatorname{Isom}\left(\mathbb{H}^{3}\right),
$$

is first-order rigid except for conjugacy. (I.e., it is infinitesimally rigid at the level of the first derivative.) The same is true if $N$ is cusped, among representations that are parabolic at the torus cusps.

Corollary 5.10 (Stated by Izmestiev [15, Sec. 1.3]). If $\Theta$ is a geometric triangulation of a closed or cusped hyperbolic 3manifold $N^{*}$, then it is first-order rigid except for motion of the non-ideal vertices.

Since we could not find a proof of Corollary 5.10 in the literature, we provide one in Section 5.4.

Proof of case 2 of Lemma 5.7. We fix the model of each tetrahedron in the upper half space model so that it has exactly six degrees of freedom, or five if one of the vertices is ideal. After ordering the vertices $v_{1}, v_{2}, v_{3}, v_{4}$, we can put vertex $v_{1}$ at $(0,0,1)$; we can put vertex $v_{2}$ directly below it (or at $(0,0,0)$, allowing it to be the ideal vertex); and we can put $v_{3}$ at a position of the form $(a, 0, b)$. We approximate the positions of the vertices approximately with rational numbers. We can then approximate the lengths and angles of each tetrahedron in the same form, as well as the first and second derivatives of the lengths and angles as a function of the main variables, the separate positions of the vertices in the ideal models of the tetrahedra.

Suppose that there are $n$ non-ideal vertices. By the implicit function theorem, any exact solution to the gluing equations for the tetrahedra can be perturbed so that some $3 n$ of the coordinates are exactly rational. Also by the implicit function theorem, some $3 n$ of the angle conditions are implied by the other angle conditions and can be omitted. Finally, the fixed coordinates and omitted angle conditions can be chosen so that the remaining system of constraints, which we can write abstractly as $f(x)=0$, has a non-singular Jacobian $D f$.

Moreover, the mapping $f$ is real analytic with an explicit formula. Thus, given an approximate solution $x_{0}$ which is within $\varepsilon$ of a true solution and $\varepsilon$ is small enough, we can majorize $\left\|(D f(x))^{-1}\right\|$ on the ball $B_{\varepsilon}\left(x_{0}\right)$ using Taylor series, to confirm equation (1).

As Lemma 5.7 addresses the hyperbolic case of Theorem 5.4, we turn to a lemma and a proposition that address the Seifert-fibered case.

Lemma 5.11. It is recursive to find an adapted triangulation of a Seifert-fibered manifold $N$ which is either closed or has torus boundary components. Hence, it is in RE to determine if it is Seifert-fibered.
Proof. We can search through triangulations until we find one that is a barycentric subdivision of a cellulation by triangular prisms. It is then easy to check whether the prisms fit together following the rules in Section 5.2.

Finally, a torus $T$ that has matching Seifert-fibered structure on both sides is not needed and is not a JSJ torus. It is easy to see this case in the proof of Theorem 5.2. The more subtle possibility is that one or both sides might have more than one Seifert fibration. Fortunately this is rare for Seifert-fibered manifolds with boundary. It is addressed by the following result.

Theorem 5.12 (Waldhausen [16, Thm. VI.17 \& Lem. VI.19]). Let $N$ be an oriented 3-manifold with non-empty boundary $\partial N$ and which has at least one Seifert fibration. Then the fibration is uniquely determined up to isotopy by its restriction to $\partial \mathrm{N}$, and is outright unique except the following cases:

1. If $N$ is a solid torus $D^{2} \times S^{1}$, then every fibration of $\partial N$ extends to a fibration of $N$ over a disk $D^{2}$ with at most one exceptional fiber.

2. If $N$ is a thickened torus $S^{1} \times S^{1} \times I$, then every fibration is a trivial circle bundle over an annulus. There is such a fibration for every rational slope in a single torus $S^{1} \times$ $S^{1}$.

3. If $N$ is a twisted I-bundle $K^{2} \ltimes I$ over a Klein bottle $K^{2}$, then it has two non-isotopic fibrations. One fibration is over a Möbius strip with Seifert data $\left\{S^{1} \ltimes I\right\}$, and one is over a disk $D^{2}$ with Seifert data $\left\{D^{2},(2,1),(2,1)\right\}$.

Proof of Theorem 5.4. We search over triangulations $\Theta$ of $M$ using stellar or bistellar moves, and decorations of them, to find an adapted triangulation as described in Section 5.2. A suitable decoration consists of distinguished spheres and thickened tori, and a reverse barycentric subdivision in each JSJ component $N$ to make triangular prisms in the Seifertfibered case and a combination of once-truncated and ordinary tetrahedra in the hyperbolic case. Within this search, we search for geometric data to describe the hyperbolic structure of each $N$ which is not Seifert-fibered. Since these are nested, infinite searches, we combine them using the RE search algorithm of Proposition 2.5. By the geometrization theorem, we will eventually find a $\Theta$ that fits the description of Section 5.2.

We examine the JSJ components to verify that all of the spheres and tori are essential and that no two are parallel. We veto $\Theta$ if it includes a Seifert-fibered solid torus (by checking that the base orbifold is a disk with at most one exceptional fiber). If all JSJ components are either hyperbolic or Seifertfibered, and if none are solid tori, then every sphere and torus is essential and no two spheres are parallel. Two distinct tori are parallel if and only if the component between them is a thickened torus; we veto this as well. We also need to veto a Seifert-fibered component homeomorphic to $P^{3} \# P^{3}$, which is the only Seifert-fibered space that is not a prime 3-manifold. This space has Seifert data $\left\{P^{2}, 0\right\}$.

Finally, we need to check that all of the tori are JSJ tori. We veto $\Theta$ if there is a torus $T$ that has Seifert-fibered components on both sides that: 
1. restrict to the same fibration of $T$; or

2. could be refibered to restrict to the same fibration of $T$.

Case 1 is easy to recognize. By Theorem 5.12 and the comments after, in case 2 we only have to consider two types of Seifert-fibered components, which can be recognized explicitly from any of their fibrations:

2a. $N \cong S^{1} \times S^{1} \times I$, or

2b. $N \cong K^{2} \ltimes I$.

Case 2a is only possible if $N$ is glued to itself to make $W$ a torus bundle over a circle, $S^{1} \ltimes\left(S^{1} \times S^{1}\right)$, because we do not allow parallel tori. The torus is needed if and only if $W$ is a Sol manifold. We can verify this case by confirming that the holonomy matrix in $\operatorname{SL}(2, \mathbb{Z})$ has distinct, real eigenvalues.

In case $2 \mathrm{~b}, N \cong K^{2} \ltimes I$ only has one torus boundary component $T$, so its refibration does not affect any other torus. In this case the fibration of $N$ may have Seifert data $\left\{S^{1} \ltimes I\right\}$ or $\left\{D^{2},(2,1),(2,1)\right\}$. The resulting binary choice may occur on one or both sides of $T$, and we veto $\Theta$ if the Seifert fibrations extend across $T$ for any of these choices.

\subsection{Proof of Corollary 5.10}

The idea of the proof is that we can convert a first-order deformation of a triangulation of $N^{*}$ to a deformation of a representation of $\rho$, in much the same way that we can convert a triangulation to $\rho$ in the first place.

Proof. In general, if $\Gamma$ is a discrete group (such as the fundamental group of a topological space) and $G$ is a Lie group, then we can describe a first-order deformation of a homomorphism $\rho: \Gamma \rightarrow G$ as a homomorphism

$$
\left(\rho, \rho^{\prime}\right): \Gamma \rightarrow G \ltimes \mathfrak{g} .
$$

Here $\mathfrak{g}$ is the Lie algebra of $G$ viewed as a group under addition, while $G \ltimes \mathfrak{g}$ is the semidirect product in which the nonnormal subgroup $G$ acts on the normal subgroup $\mathfrak{g}$ by conjugation. Also, $\left(\rho, \rho^{\prime}\right)$ should reduce to $\rho$ under the quotient map

$$
\pi: G \ltimes \mathfrak{g} \rightarrow G .
$$

Note that $G \ltimes \mathfrak{g}$ is also the total space of the tangent bundle $T G$. In other words, the extension $\rho^{\prime}$ is a choice of a tangent vector $\rho^{\prime}(g) \in T_{\rho(g)} G$ for every $g \in \Gamma$, such that the pairs $\left(\rho(g), \rho^{\prime}(g)\right)$ together make a group homomorphism.

Suppose that $\Gamma=\Gamma_{1}(X)$ is the fundamental group of a based CW complex $X$. Then we can model $\rho$ (non-uniquely) as a non-commutative cellular cocycle $\alpha \in C^{1}(X ; G)$. Given $\rho$, we can likewise model the extension $\rho^{\prime}$ (also non-uniquely) as a commutative cocycle $\alpha^{\prime} \in C^{1}(X ; \mathfrak{g})$, where here $\mathfrak{g}$ is a coefficient system twisted by $\alpha$.

Now let $X=N$, where $N$ has a cellulation $\Theta$ that comes from a closed or cusped hyperbolic structure on $N^{*}$ and a geometric triangulation $\Theta^{*}$. If $N$ is cusped and $\Theta^{*}$ is a semi-ideal triangulation, then we make $\Theta$ by truncating the ideal vertices of $\Theta^{*}$. We then want to make a cocycle $\alpha$ from $\gamma$. To do this, we first choose a specific isometry $\tilde{N}^{*} \cong \mathbb{H}^{3}$. Then we choose an orthonormal tangent frame at each vertex of $\Theta$. Given an edge $e \in \Theta$, we let $\alpha(e)$ be the element of $G=\operatorname{Isom}^{+}\left(\mathbb{H}^{3}\right)$ that takes the tail $\tilde{v}$ of a lift $\tilde{e}$ to the head $\tilde{w}$, and takes the lifted frame of $\tilde{v}$ to the lifted frame of $\tilde{w}$. If $N^{*}$ is cusped, then we require that each truncation edge in $N$ is assigned a parabolic element that fixes the corresponding ideal vertex in $N^{*}$.

In this setting, Theorem 5.9 says that $H^{1}(N ; \mathfrak{g})=0$ in the closed case and $H^{1}(N, \partial N ; \mathfrak{g}, \mathfrak{p})=0$ in the cusped case, where $\mathfrak{g}$ is the parabolic Lie subalgebra of $\mathfrak{g}$. The theorem is typically proved using de Rham cohomology rather than cellular cohomology, but these models of cohomology are isomorphic as usual. More explicitly, every 1-cocycle $\alpha^{\prime}=\delta \beta$, where $\beta$ is an $\mathfrak{g}$-valued 0 -cochain on the vertices of $\Theta$.

Finally, suppose that $\gamma^{\prime}$ is a first-order deformation of the hyperbolic structure $\gamma$ of $\Theta^{*}$ that satisfies the first derivative of the gluing equations. Then we can lift $\gamma^{\prime}$ to a cocycle $\alpha^{\prime}$ (non-uniquely) in the same way that $\gamma$ lifts to $\alpha$. Then Theorem 5.9 provides $\beta$, and $\beta$ descends to a first-order motion of the vertices of $\Theta^{*}$ that induces the deformation $\gamma^{\prime}$.

\section{HOMEOMORPHISM IS RECURSIVE}

The goal of this section is to prove Theorem 1.1 in this section, postponing only the proof of Theorem 6.1 below until Section 7.

\subsection{Connected sums}

If $M_{1}$ and $M_{2}$ are two closed, oriented 3-manifolds given by triangulations, then by Theorem 5.4, we know the direct sum decompositions of each one into prime 3-manifolds. These summands can be freely permuted and can only be matched in finitely many ways. If we search over the ways to match them, we then reduce the oriented homeomorphism problem $M_{1} \stackrel{?}{\cong} M_{2}$ to the oriented homeomorphism problem $W_{1} \stackrel{?}{\cong} W_{2}$ for prime summands $W_{1}$ and $W_{2}$. To review, each summand $W_{k}$ inherits an orientation from its parent $M_{k}$; in the reverse direction, there is no ambiguity in forming an oriented connected sum.

\subsection{One JSJ component}

We switch to the other end of geometric decompositions to analyze a single pair of JSJ components $N_{1} \subseteq W_{1} \subseteq M_{1}$ and $N_{2} \subseteq W_{2} \subseteq M_{2}$. We are interested not only in the isomorphism problem, but also in the effect of the mapping class group of a component $N$ on the boundary $\partial N$.

Theorem 6.1. Suppose that $N$ is an oriented, hyperbolic JSJ summand such that $N^{*}$ is either closed or cusped. Then the mapping class group of $N$ is its isometry group. It is a finite group and its computation is recursive. If $N_{1}$ and $N_{2}$ are two 
such manifolds, then they are homeomorphic if and only if they are isometric, and recognizing this condition is recursive.

Again, we will prove Theorem 6.1 in Section 7. Note that if $N$ is hyperbolic and has torus boundary components, then each such component inherits a Euclidean structure from the hyperbolic structure on $N^{*}$.

Suppose instead that $N$ is Seifert-fibered (and, as usual, oriented). Then in the direct sense the automorphism problem only matters for Theorem 1.1 when the JSJ graph is non-trivial and thus $N$ has boundary. However, we will learn the relevant automorphism properties from an associated closed Seifertfibered space.

Lemma 6.2. Let $N$ be a closed, oriented 3-manifold which is decorated with a Seifert fibration with Seifert data

$$
\left\{F, b,\left(a_{1}, b_{1}\right),\left(a_{2}, b_{2}\right), \ldots,\left(a_{n}, b_{n}\right)\right\} .
$$

Then:

1. The exceptional fibers of $N$ are freely permutable by automorphisms of the Seifert fibration, provided that the permutation preserves the orbifold number $a_{k} \geq 2$ and the residue $b_{k} \in \mathbb{Z} / a_{k}$ of each exceptional fiber.

2. Any finite set of regular fibers is freely permutable.

3. If the base $F$ is orientable, then $N$ has a homeomorphism that inverts all fibers together, but they cannot be inverted separately.

4. If the base $F$ is non-orientable, then given two disjoint finite sets $A, B \subseteq F, N$ has a homeomorphism that inverts the fibers over $A$ in place and fixes the fibers over B.

Proof. Cases 1, 2, and 4 can all be established by isotopies of $F$ that move points that correspond to the distinguished fibers. In case 4 , using the hypothesis that $F$ is non-orientable, we can move a point $p \in A$ around an orientation-reversing loop in $F$ that stays away from $B$ and from the rest of $A$.

Meanwhile in case 3, the fibration itself is orientable, which means that an orientation of any one fiber induces a canonical orientation of all fibers. On the other hand, Seifert's construction of the fibration via vertical Dehn surgery on $F \times S^{1}$ is invariant with respect to inverting the $S^{1}$ factor and simultaneously applying an orientation-reversing homeomorphism to $F$.

We also need the counterpart to Theorem 5.12 for closed Seifert-fibered spaces.

Theorem 6.3 (Waldhausen [16, Thm. VI.17]). If $N$ is a closed, oriented Seifert-fibered 3-manifold, then its Seifert fibration is unique up to homeomorphism except in the following cases:

1. A Seifert-fibered space with base $S^{2}$ and at most two exceptional fibers is either a lens space $L(m, n), S^{2} \times S^{1}$, or $S^{3}$
2. A Seifert-fibered space with base $P^{2}$ and at most one exceptional fiber is either a lens space $L(4, n)$, a prism space $R(m, n)$, or $P^{3} \# P^{3}$.

3. The space with Seifert data

$$
\left\{S^{2}, b,(2,1),(2,1),\left(a_{1}, b_{1}\right)\right\}
$$

is a prism space $R(m, n)$.

4. The twisted bundle $K^{2} \ltimes S^{1}$ which is the double of $K^{2} \ltimes I$ has the double of its two fibrations, namely the Seifert data $\left\{K^{2}, 0\right\}$ and the Seifert data

$$
\left\{S^{2}, 0,(2,1),(2,1),(2,1),(2,1)\right\} .
$$

Theorem 6.3 comes with simple formulas for which lens space or prism space is obtained, which we omit. In particular, the answer is recursive and (as we will later want) elementary recursive.

\subsection{The JSJ graph}

If $W$ is a prime 3-manifold, then its JSJ decomposition is modelled by a labelled graph $\Gamma$, whose vertices represent JSJ components and whose edges represent connecting tori. Each vertex is labelled by the homeomorphism type of its component, which is either Seifert-fibered or hyperbolic. In addition, each edge is decorated with gluing data and peripheral data which will be described precisely in the proof of Theorem 1.1 below.

Remark. This graph structure inspired the term graph manifold for a prime 3-manifold whose JSJ components are all Seifert-fibered [51]. This terminology is standard but ironic, since geometrization shows that the same graph concept is important for all prime 3-manifolds.

The labelled graph $\Gamma$ is an invariant of $W$, which at first glance may seem like a complete invariant, provided that the homeomorphism problem for each JSJ component is recursive. However, it is not that simple, because we have to know the allowed permutations of the torus boundary components of a JSJ component $N$, and the allowed homeomorphisms of each torus boundary component. Finally, we need to deal with the special case that $N$ is either $K^{2} \ltimes I$ or $S^{1} \times S^{1} \times I$ and thus has more than one Seifert fibration.

Proof of Theorem 1.1. (Proof using case 1 of Lemma 5.7.) As explained in Section 6.1, it suffices to solve the homeomorphism problem $W_{1} \stackrel{?}{\cong} W_{2}$ for prime 3 -manifolds $W_{1}$ and $W_{2}$. The proof is divided into three steps. In steps 1 and 2, we let $W$ be a prime 3-manifold and let $\Gamma$ be its JSJ graph. It is recursive to calculate $\Gamma$ and the isomorphism types of its vertices.

Step 1: We address the cases in which a JSJ component of $W$ has more than one Seifert fibration. If any component is a $K^{2} \ltimes I$, then its two fibrations (described in Theorem 5.12) are inequivalent; we choose one of them and use it for every 
occurrence of $K^{2} \times I$ in $W$. If a JSJ component $N$ is a thickened torus, then as in the proof of Theorem $5.4, W$ is a Sol manifold and a torus bundle over a circle, $S^{1} \ltimes\left(S^{1} \times S^{1}\right)$. In this case the homeomorphism type of $W$ is given by a pair of conjugacy classes in $\operatorname{SL}(2, \mathbb{Z})$, one for each orientation of the base circle. Recall that the conjugacy classes in $\operatorname{SL}(2, \mathbb{Z})$ can be classified with the aid of the isomorphism

$$
\operatorname{PSl}(2, \mathbb{Z}) \cong C_{2} * C_{3}
$$

If $g \in \mathrm{SL}(2, \mathbb{Z})$ has non-zero trace (which it does if $W$ is Sol), then its conjugacy class is given by the sign of its trace and its reduced cyclic word in $C_{2} * C_{3}$.

Step 2: We suppose that $W$ is not a Sol torus bundle over a circle. If $T$ is a JSJ torus in $W$ and one side of $T$ is a hyperbolic component $N$, then $T$ inherits a Euclidean structure which we can normalize to have area 1. This Euclidean structure can be described by a quadratic form $Q$ on the first homology $H_{1}(T)=H_{1}(T ; \mathbb{Z})$, where $Q(c)$ is the square of the minimum length of $c \in H_{1}(T)$. Moreover, the coefficients of $Q$ are real algebraic numbers computable from the geometry of $N$. On the other hand, if $N$ is Seifert-fibered, then the induced fibration of $T$ selects a line in $H_{1}(T)$, by which we mean a rank-one subgroup $L \subseteq H_{1}(T)$ with a torsion-free quotient $H_{1}(T) / L$.

Since $T$ has two sides, it is then decorated by a pair of quadratic forms on $H_{1}(T)$, or a quadratic form and a line, or a pair of lines. In the third case when both sides of $T$ are Seifert-fibered, the fibrations must be mismatched at $T$, so the two lines $L_{1}, L_{2} \subseteq H_{1}(T)$ must be distinct. Hence they constitute a rational line basis in the sense that

$$
H_{1}(T ; \mathbb{Q})=\left(L_{1} \otimes \mathbb{Q}\right) \oplus\left(L_{2} \otimes \mathbb{Q}\right) .
$$

We also obtain a rational line basis in the second case, when one side is Seifert-fibered and produces a line $L_{1}=L$, and the other side is hyperbolic and produces a quadratic form $Q$. In this case, there exist a finite set of pairs of homology classes $\pm c \in H_{1}(T) \backslash L_{1}$ that minimize $Q(c)$. If there is only one such pair, we let $L_{2}$ be the line generated by $\pm c$. If there is more then one, we let $L_{2}$ be the line generated by the first such pair in the clockwise direction from $L_{1}$.

Note that each possible decoration of $T$ induced by the geometry on both of its sides has a finite stabilizer in the oriented mapping class group $\operatorname{SL}\left(H_{1}(T)\right) \cong \operatorname{SL}(2, \mathbb{Z})$ of $T$. If we order the two sides of $T$, then the stabilizer usually has two elements; in rare cases it is a cyclic group of order 4 or 6.

If $N$ is a Seifert-fibered component of $W$, then each of its torus boundary components is decorated by a rational line basis. We can thus make a closed Seifert-fibered space $\hat{N}$ by collapsing a circle fibration of each component $T \subseteq \partial N$ that represents the opposite line in $H_{1}(T)$, the one that does not come from $N$ itself. Each torus component of $\partial N$ becomes a distinguished fiber in $\hat{N}$ which may be either regular or exceptional.

Step 3: Suppose that $W_{1}$ and $W_{2}$ are two prime, closed, oriented 3-manifolds. If they do not have any JSJ tori, then they are both Seifert-fibered, and we can use Theorem 6.3 to tell if they are the same. If they are both Sol torus bundles, we can use the algorithm in step 1 to determine if they are homeomorphic.

Otherwise we can assume that $W_{1}$ and $W_{2}$ have non-trivial JSJ graphs $\Gamma_{1}$ and $\Gamma_{2}$, and that each JSJ torus has a canonical decoration as described in step 2. To determine if $W_{1}$ and $W_{2}$ are homeomorphic, we search over graph isomorphisms $f: \Gamma_{1} \rightarrow \Gamma_{2}$. For every pair of $T_{1} \subseteq W_{1}$ and $T_{2} \subseteq W_{2}$ that are matched by $f$, we search over mapping classes that preserve the canonical decorations of $T_{1}$ and $T_{2}$. In the innermost part of the search, we want to calculate whether the homeomorphisms of the JSJ tori extends to each matched pair of JSJ components $N_{1} \subseteq W_{1}$ and $N_{2} \subseteq W_{2}$. If $N_{1}$ and $N_{2}$ are hyperbolic, then we can use Theorem 6.1 to determine if the homeomorphism $\partial N_{1} \cong \partial N_{2}$ extends. If they are both Seifertfibered, then we can use Lemma 6.2 to determine whether the corresponding closed Seifert-fibered manifolds $\hat{N}_{1}$ and $\hat{N}_{2}$ have a homeomorphism that extends the given homeomorphism $\partial N_{1} \cong \partial N_{2}$. Note that we can employ Lemma 6.2 because any relevant homeomorphism $N_{1} \cong N_{2}$ preserves the fibration at the boundary, and is thus isotopic to a fibrationpreserving homeomorphism by Theorem 5.12.

Proof of Theorem 1.1. (Proof using case 2 of Lemma 5.7.) If the geometric data of each hyperbolic JSJ component $N$ of a summand $W$ is described with computable real numbers rather than real algebraic numbers, then the induced Euclidean structure on a JSJ torus $T \subseteq \partial N$ is only given by a convergent sequence of approximations. Thus, it is not possible to definitively calculate the isometries of $T$ or the shortest cycles, as expressed with the quadratic form $Q(c)$. However, all nonisometries and all non-zero classes in $H_{1}(T)$ that are not shortest are eventually revealed. This yields an algorithm in coRE for the homeomorphism problem $M_{1} \stackrel{?}{\cong} M_{2}$, which is enough to show that the problem is recursive per the discussion at the beginning of Section 7 .

\section{PROOFS OF THEOREM 6.1}

In this section we will give several proofs of Theorem 6.1. Recall that Corollary 3.4 says that the existence of a PL homeomorphism $N_{1} \cong N_{2}$ is in RE; it is also easy to check whether it preserves orientation. So, by Proposition 2.3, it suffices to show that homeomorphism is in coRE, although only one of the proofs will make use of this directly. By a similar argument, finding elements in the mapping class group of a single $N$ is in RE; the remaining task is an algorithm to show that the list is complete.

Recall that if $N$ has boundary, then its interior $N^{*}$ is cusped and has a semi-ideal triangulation $\Theta^{*}$. In this case, $\Theta$ is a cellulation in which semi-ideal tetrahedra are once truncated. We want to geometrize the truncation that produces $\Theta$. We consider a horospheric truncation which is almost but not quite unique, with the following three properties:

1. The horosphere sections lie entirely within the semiideal tetrahedra of $\Theta^{*}$, and therefore do not intersect each other. 
2. For some common integer $n$, every horospheric torus has area $2^{-n}$.

3. We do not use the smallest value of $n$ that satisfies conditions 1 and 2.

For convenience, we let $N^{*}=N$ and $\Theta^{*}=\Theta$ if $N$ is closed.

Some of the proofs make use of the following lemma.

Lemma 7.1. It is recursive to obtain a lower bound in the injectivity radius of $N$ and $\Theta$.

First proof. Suppose first that $N$ is closed. For each vertex $v \in \Theta$, let $U_{v}$ be the open star of $\Theta$ containing $p$. Then the collection $\left\{U_{v}\right\}$ is a finite open cover of $N$. It follows just from topology that there is some radius $\varepsilon$ such that every ball of radius $\varepsilon$ is contained in some $U_{v}$. For an explicit calculation, let $\Theta^{\prime}$ be a barycentric subdivision of $\Theta$, and for each $v \in \Theta$, let $X_{v}$ be the closed star of $v \in \Theta^{\prime}$; then the sets $X_{v}$ are a closed cover. We can calculate or bound the distance from $X_{v}$ to $N \backslash$ $U_{w}$ for some $w \in \Theta$ with $X_{v} \subseteq U_{w}$. The minimum of all of these distances is thus a lower bound $\varepsilon$ for the injectivity radius.

Second proof. In general we use the notation $B(p, r)$ for a hyperbolic ball of radius $r$ centered at $p$.

Let $r$ be the exact injectivity radius of $N$, and let $p$ be a point on a closed geodesic of $N$ of length $2 r$. Then $p \in \Delta$ for some cell $\Delta \in \Theta$, and we can let $\ell$ be an upper bound of the diameter of $\Delta$. Then in the universal cover

$$
\tilde{N} \subseteq \tilde{N}^{*} \cong \mathbb{H}^{3},
$$

we obtain that at least $1 / 2 r$ lifts of $\Delta$ intersect $B(p, 1)$, and thus at least this many copies of $\Delta$ are contained in $B(p, \ell+1)$. Thus

$$
\frac{1}{2 r} \leq \frac{\operatorname{Vol}(B(p, \ell+1))}{\operatorname{Vol}(\Delta)},
$$

hence

$$
r>\frac{\operatorname{Vol}(\Delta)}{2 \operatorname{Vol}(B(p, \ell+1))} .
$$

We can calculate an upper bound of this form, if necessary using a lower bound for the numerator and an upper bound for the denominator, for every cell in $\Theta$, since we do not know the position of the shortest geodesic loop in advance.

Third proof. This proof is a variation of the second proof using the entire diameter and volume of $N$. Jørgensen and Thurston proved that the set of possible volumes of $N^{*}$ is wellordered. In particular, there is one of least volume, so there is some constant $c>0$ such that

$$
\operatorname{Vol}\left(N^{*}\right)>c
$$

Our construction of the geometry of $N$ spares more than half of the volume of $N^{*}$, so

$$
\operatorname{Vol}(N)>\frac{\operatorname{Vol}\left(N^{*}\right)}{2}>\frac{c}{2}=c^{\prime} .
$$

We can obtain an upper bound $\ell$ on the diameter of all of $N$ by adding bounds on the diameters of the cells in $\Theta$. Then, we let $D$ be a convex fundamental domain for $N$; it has the same volume and diameter at most $2 \ell$. Thus we obtain an estimate similar to (2), but more robust:

$$
r>\frac{\operatorname{Vol}(D)}{2 \operatorname{Vol}(B(p, \ell+1))}>\frac{c^{\prime}}{2 \operatorname{Vol}(B(p, \ell+1))} .
$$

Remark. Without an explicit bound on least-volume closed or cusped hyperbolic manifold, the third proof has the unusual feature of non-constructively proving that an algorithm exists, i.e., without fully stating the algorithm. Meyerhoff [30] established the first lower bound

$$
\operatorname{Vol}(N) \geq \frac{2}{5^{4}}
$$

in the closed case. In the same paper, he and Jørgensen established

$$
\operatorname{Vol}\left(N^{*}\right) \geq \frac{\sqrt{3}}{4} \Longrightarrow \operatorname{Vol}(N) \geq \frac{\sqrt{3}}{8}
$$

in the cusped case. The exact minimum values are now known [9].

First proof of Theorem 6.1. This proof is similar to one given by Scott and Short [43]. We assume geometric triangulations $\Theta_{1}^{*}$ and $\Theta_{2}^{*}$ of $N_{1}^{*}$ and $N_{2}^{*}$.

If $N_{1}^{*}$ and $N_{2}^{*}$ are homeomorphic and therefore isometric, then we can intersect the tetrahedra of $\Theta_{1}^{*}$ and $\Theta_{2}^{*}$ to make a tiling of $N_{1} \cong N_{2}$ by various convex cells with 8 or fewer sides; we can then take a barycentric subdivision to make tetrahedra. We thus obtain a mutual refinement $\Theta_{3}$ of $\Theta_{1}$ and $\Theta_{2}$. If we can bound the complexity of $\Theta_{3}$, then we can find it with a finite search or show that it does not exist, rather than using stellar or bistellar moves in both the up and down directions.

Let $\Delta_{1} \in \Theta_{1}^{*}$ and $\Delta_{2} \in \Theta_{2}^{*}$ be two tetrahedra in the separate triangulations. In the universal cover $\tilde{N}_{1}^{*}$, they can only intersect in a single cell with at most 8 sides. In $N_{1}^{*}$ itself they can intersect many times; however, only as often as different lifts of $\Delta_{1}$ intersect one fixed lift of $\Delta_{2}$. If $\Delta_{1}$ and/or $\Delta_{2}$ are semiideal, then their lifts intersect if and only if their truncations do. There is a recursive volume bound on the number of possible intersections by the same argument as the second proof of Lemma 7.1.

Having bounded the necessary complexity of a mutual refinement $\Theta_{3}$, we can now search over separate refinements $\Theta_{3}$ of $\Theta_{1}$ and $\Theta_{4}$ of $\Theta_{2}$ using Proposition 3.5, and look for an orientating-preserving simplicial isomorphism $\Theta_{3} \cong \Theta_{4}$. The same method can be used to calculate the mapping class group of a single $N$.

Second proof. Suppose that $X_{1}$ and $X_{2}$ are two compact metric spaces, and suppose that for each $\varepsilon>0$ we have a way to make finite $\varepsilon$-nets $S_{1}$ and $S_{2}$ for $X_{1}$ and $X_{2}$, and calculate or approximate all distances within $S_{1}$ and within $S_{2}$. If $X_{1}$ and $X_{2}$ are isometric, then there is a function $f: S_{1} \rightarrow S_{2}$ that changes distances by at most $2 \varepsilon$. On the other hand, if there is such a function for every $\varepsilon$, then $X_{1}$ and $X_{2}$ must be isometric. 
In our case, we let $X_{k}=N_{k}$, where we make sure to use the same truncation area $2^{-n}$ to geometrize $N_{1}$ and $N_{2}$ given the geometries of $N_{1}^{*}$ and $N_{2}^{*}$. We calculate a common lower bound $\delta$ on the injectivity radius.

We can choose some convenient coordinates inside each cell $\Delta \in \Theta_{k}$. We then have the ability to calculate geodesic segments in $N_{k}$ that are made of geodesic segments in the separate tetrahedra. If $\Delta$ is truncated, then the geodesic segment might hug the truncation boundary for part of its length, but it still has a finite description. Without more work, we don't know which of these geodesics are shortest geodesics. However, if a geodesic is shorter than $\delta$, then it is shortest. Taking $\delta \gg \varepsilon \rightarrow 0$, we can make $\varepsilon$-nets of both $N_{1}$ and $N_{2}$ and look for approximate isometries between these $\varepsilon$-nets; it suffices to check distances below the fixed value $\delta$.

More explicitly, we can use the covering by open stars $S_{v}$ in the first proof of Lemma 7.1. There is a $\delta$ such that if $d(x, y)<$ $\delta$, then $x$ and $y$ and even the connecting short geodesic are all in some open star.

This algorithm does not by itself ever prove that $N_{1}$ and $N_{2}$ are isometric, only that they aren't. Thus it shows that the homeomorphism problem is in coRE. This is good enough by Proposition 2.3 and Corollary 3.4.

The algorithm also does not by itself determine whether the isometry is orientation-preserving. However, this is very little extra work. Given $\varepsilon \ll \delta$ and given $\varepsilon$-nets $S_{1} \subseteq N_{1}$ and $S_{2} \subseteq N_{2}$, we can let $p_{1}, p_{2}, p_{3}, p_{4}$ be 4 points in $S_{1}$ that lie in a ball of radius $\delta / 2$ and that make an approximately regular tetrahedron $\Delta$. If $f: S_{1} \rightarrow S_{2}$ is an approximate isometry, then we can check whether $f$ flips over $\Delta$. If no orientationpreserving isometry exists, then when $\varepsilon$ is small enough, either $f$ will cease to exist or $\Delta$ will be flipped over.

We can use similar methods to find the mapping class group of a single $N$, since by Mostow rigidity it is also the isometry group of $N$. We assume that $N$ has boundary, which is technically short of the full generality of Theorem 6.1, but enough to prove Theorem 1.1. Just as with the method to check whether and approximate $f$ preserves orientation, we can when $\varepsilon$ is small enough compute the effect of $f$ on $H_{1}(\partial N)$, which determines which isometry is close to $f$ (if any).

Third proof. In this proof, we restrict attention to case 1 of Lemma 5.7 and thus work over the ring $\hat{\mathbb{Q}}$ of real algebraic numbers. We assume real algebraic coordinates for $\mathbb{H}^{3}$ and for its isometry group $\operatorname{Isom}^{+}\left(\mathbb{H}^{3}\right)$; for example we can take $\mathbb{H}^{3}$ to be the set of positive, unit timelike vectors in 3+1-dimensional Minkowski geometry, and we can take $\operatorname{Isom}^{+}\left(\mathbb{H}^{3}\right)=\operatorname{ISO}(3,1)$. We again assume that $N_{1}$ and $N_{2}$ are made from $N_{1}^{*}$ and $N_{2}^{*}$ using a common truncation area $2^{-n}$.

We assume geometric triangulations $\Theta_{1}^{*}$ and $\Theta_{2}^{*}$ of $N_{1}^{*}$ and $N_{2}^{*}$ with real algebraic descriptions. Using these triangulations, we can find finite, open coverings of $N_{1}$ and $N_{2}$ by metric balls $B(p, \varepsilon)$, where each point $p$ has a real algebraic position and the common radius is (a) also real algebraic, and (b) less than half of the injectivity radius of $N_{1}$ and $N_{2}$. Then we can give each ball the same algebraic coordinates as $\mathbb{H}^{3}$, and we can also calculate the relative position of every pair of balls as some element in $\operatorname{Isom}^{+}\left(\mathbb{H}^{3}\right)$. In other words, we obtain atlases of charts for $N_{1}$ and $N_{2}$ using the $\operatorname{Isom}^{+}\left(\mathbb{H}^{3}\right)$ pseudogroup. In fact, everything is constructed in the subgroup and sub-pseudogroup with real algebraic matrix entries.

If there is an isometry between $N_{1}$ and $N_{2}$, then their atlases combine into a larger atlas. There are only finitely many possible patterns of intersection between the balls of $N_{1}$ and the balls of $N_{2}$. For each such pattern, we obtain a finite system of algebraic equalities and inequalities, which says first that the intersection pattern is what is promised, and second that the gluing maps between the atlases are consistent. Theorem 2.8 then says that it is recursive to determine whether this system of equations has a solution. Since we work in the group Isom ${ }^{+}\left(\mathbb{H}^{3}\right)$, we are looking only for orientationpreserving isometries.

\section{HOMEOMORPHISM IS IN ER}

We will use the basic fact that a finite composition of ER functions is in ER. In other words, if an algorithm has a bounded number of stages that expand its data by an exponential amount or otherwise by an ER amount, then it is still in ER.

\subsection{The outer proof}

In this section we will prove Theorem 1.2. The proof is a combination of the proof of Theorem 1.1 together with several computational improvements. We summarize these computational improvements in this section by stated some supporting theorems which we will prove ourselves (or prove by citation) with two main supporting tools. The first tool is normal surface theory, which we can use to find essential spheres and tori and Seifert fibrations. Note that Jaco, Letscher, and $\mathrm{Ru}-$ binstein [17] sketched a similar approach. The second tool is an ER version of Theorem 2.8 [10], which we use to bound the complexity of a geometric triangulation of a hyperbolic manifold, and the complexity of recognizing small Seifert-fibered spaces.

Theorem 8.1. It is in ER to find and triangulate the prime summands $\{W\}$ of a closed, oriented 3-manifold $M$, to find and triangulate the JSJ components $\{N\}$ within each prime summand $W$, to find their JSJ graph $\Gamma$, and to recognize which components $N$ are Seifert-fibered and find their fibrations.

We will prove most of Theorem 8.1 in Section 8.2 using normal surface theory. Note that Jaco, Letscher, and Rubinstein [17] sketched ideas that are similar to our proof.

Theorem 8.1 also has one lingering case which is more difficult. Recall that a Seifert-fibered space is small if it is nonHaken (and therefore closed).

Theorem 8.2. Recognizing small Seifert-fibered spaces is in ER.

We will prove Theorem 8.2 in Section 8.4 using a combination of normal surface theory and algebraic methods. 
Note that Li [27] shows that recognizing small Seifert-fibered spaces with infinite $\pi_{1}$ is recursive, and his algorithm should be elementary recursive. However, we will use a different approach for this part of the theorem. Li also addresses the finite $\pi_{1}$ case in two different ways. Without assuming geometrization (which was still open at the time), he cites work of Rubinstein and Rannard-Rubinstein on small Seifert-fibered spaces. He also outlines a simplified argument for the finite $\pi_{1}$ caes that depends on geometrization; we give a detailed argument which is in a similar spirit.

Theorem 8.3. If a compact, oriented 3-manifold $N$ has a closed or cusped hyperbolic structure, then it is in ER to find a geometric triangulation and specify its geometric data with algebraic numbers. The isomorphism and automorphism problems are also both in ER.

We will prove Theorem 8.3 in Section 8.5 using both Mostow rigidity and methods from algebraic geometry.

Proof of Theorem 1.2. We consider each stage of the proof of Theorem 1.1 in turn. The proof begins with a geometric recognition of a single closed, oriented 3-manifold $M$ in Theorem 5.4. This is not elementary recursive as described, but we can replace it with Theorem 8.1 to find the direct sum and JSJ decomposition. We can then apply Theorem 8.3, which is an ER version of Lemma 5.7, to calculate the hyperbolic structure of each hyperbolic summand $N$. This includes a description of the Euclidean structure of each torus component of $\partial N$.

Finally given two closed, oriented 3-manifolds $M_{1}, M_{2}$, we first decompose them into summands. For each bijection among the summands, we want to calculate $W_{1} \stackrel{?}{\cong} W_{2}$ for each pair of matching summands. This is a calculation with JSJ graphs which is done in Section 6.3 to complete the proof of Theorem 1.1, and this part is already elementary recursive.

\subsection{Normal surfaces}

Let $M$ be a compact 3-manifold with triangulation $\Theta$. Recall that a normal surface $S \subseteq M$ intersects each tetrahedron $\Delta \in \Theta$ in 7 types of elementary disks, namely 4 types of triangles and 3 types of quadrilaterals. The surface $S=S_{v}$ is given by a vector $v \in \mathbb{Z}_{\geq 0}^{7 t}$ that lists the number of each type of elementary disk. If $v$ is such a vector, then $S_{v}$ is embedded (and uniquely defined) provided that it only uses at most one type of quadrilateral in each tetrahedron. After specifying which type of quadrilateral is allowed in each tetrahedron, the normal surface equations then have a polytopal cone

$$
C \subseteq \mathbb{Z}_{\geq 0}^{5 t} \subseteq \mathbb{Z}_{\geq 0}^{7 t}
$$

of solutions. We define a fundamental surface $S_{v}$ is one whose vector $v \in C$ is not the sum of two other solutions in $C$. If $S_{v}$ is non-orientable, then $S_{2 v}$ is its orientable double cover and we call it fundamental as well.
Lemma 8.4 (Haken). The number of elementary disks in a fundamental surface in $M$ is bounded above by an exponential in $t$. (Thus it is elementary recursive.)

We can represent a normal surface $S$ by listing all triangles and quadrilaterals in order in each tetrahedron $\Delta \in \Theta$. It is then easy to separate $S$ into connected components and calculate the topology of each component. This is exponentially inefficient compared to algorithms such as Agol-Hass-Thurston [2], but it has no effect on whether the resulting algorithm is in ER.

We define a complete set of essential 2-spheres in a 3manifold $M$ is a collection $C$ such that cutting $M$ along each 2-sphere in $M$ and capping off the resulting boundary components produces irreducible 3-manifolds. Likewise a complete set of essential disks is a collection $C$ of properly embedded disks which are not boundary parallel, such that the compression of every disk in $C$ renders $M$ boundary-incompressible.

Theorem 8.5 (Jaco-Tollefson). Let $M$ be a compact, oriented, triangulated 3-manifold. Then:

1. $M$ has a collection of disjoint, fundamental surfaces which form a complete set of essential 2-spheres [18, Thm. 5.2].

2. If $M$ has no essential 2-spheres, then it has a collection of disjoint, fundamental surfaces which form a complete set of essential disks [18, Thm. 6.2].

3. If $M$ has no essential 2-spheres or disks, and it has an essential torus, then it has one which is fundamental [18, Cor. 6.8].

4. If $M$ has no essential 2-spheres or disks, and it has an essential annulus, then it has one which is fundamental [18, Cor. 6.8].

Actually Jaco and Tollefson show that each type of surface described in Theorem 8.5 is a vertex surface, which is a special case of a fundamental surface. Also case 1 of Theorem 8.5 is stated for closed manifolds, but the proof is the same for manifolds with boundary. Finally, given Lemma 8.4, the surfaces produces by Theorem 8.5 all have an elementary recursive bound on their size.

We will use the following variation of Theorem 8.5.

Theorem 8.6 (Hass-K. [14]). If $M$ is a closed, oriented 3manifold with a triangulation $\Theta$, then it has a collection of disjoint normal surfaces which form a complete set of essential spheres and tori, such that the total number of elementary disks is bounded above by an exponential in $t$.

Briefly, Theorem 8.6 uses a generalization of the normal surface equations which we call the disjoint normal surface equations. (They are similar to the crushed triangulation technique defined by Casson [17].) They are equations for a normal surface $S$ which is disjoint from a fixed normal surface $R \subseteq M$. To prove Theorem 8.6, we find each surface $S$ or $T$ sequentially as a fundamental surface, relative to the union of previous surfaces. 
Theorem 8.7 (Rubinstein [40], Thompson [48]). Recognizing the 3-sphere $S^{3}$ is in $\mathrm{ER}$.

The proof of Theorem 8.7 uses a variant known as almost normal surfaces that are allowed one exceptional intersection with a tetrahedron that is either an octagon, or a triangle and a quadrilateral with a connecting annulus. The original papers only claim a recursive algorithm, but the algorithm is based on normal surface theory. In fact, the proof also uses disjoint normal surface equations. Schleimer [42] also refines the Rubinstein-Thompson algorithm to show that 3 -sphere recognition is in the complexity class NP, which is a much better bound than just ER.

Proof of Theorem 8.1. As a first step, we check whether $M \cong$ $S^{3}$ using Theorem 8.7. If not, we search over collections $C$ of normal surfaces in $M$ with a suitable elementary recursive complexity bound in order to find a set of surfaces that meets the conclusion of case 1 of Theorem 8.5. To test whether a given collection $C$ is one that we want, we first calculate whether each surface in it is a 2 -sphere. Then we can cut along all of the spheres (and retriangulate) and cap them to make a multiset of summands of $M$. For each non-separating sphere, we create a separate $S^{2} \times S^{1}$ summand. What remains is a putative prime factorization $\{W\}$, but we must check whether the summands are irreducible and not $S^{3}$. We can use Theorem 8.7 to check that no summand $W$ is $S^{3}$. If not, then we can again use case 1 of Theorem 8.5 to look for an essential 2 -sphere in $W$, and again use Theorem 8.7 to check whether it is essential.

For each summand $W$, we similarly search for a collection $C$ that meets the conclusion of Theorem 8.6. We can check that each surface in $C$ is a torus. We can then cut $W$ along $C$ to make a putative decomposition of $W$ into atoroidal components $\{Q\}$. We have the following possibilities for each component $Q$ :

1. $Q$ has an essential disk, which necessarily cuts it into a ball. In this case, $Q \cong S^{1} \times D^{2}$ is a solid torus.

2. $Q$ has no essential disk, but it has a separating essential annulus that cuts it into two solid tori. In this case $Q$ fibers over a disk with two exceptional fibers.

3. $Q$ does not have a separating essential annulus, but it does have a non-separating essential annulus that cuts it into a solid torus. In this case $Q$ fibers over an annulus or a Möbius strip with at most one exceptional fiber.

4. $Q$ has a separating annulus that cuts it into two thickened tori. In this case $Q \cong S^{1} \times F$, where $F$ is a pair of pants.

5. $Q$ has an essential torus, specifically an incompressible torus which is not boundary-parallel.

6. $Q=W$ is closed and has no essential torus. In this case $Q$ is either hyperbolic or small Seifert-fibered.

7. $Q$ has boundary, and it has no essential disk, annulus, or torus. In this case, $Q$ is hyperbolic.
To see that this is an exhaustive list, we recall that if $Q$ is Seifert-fibered with boundary and is atoroidal, then with one exception its orbifold base is planar, and the total number of boundary circles plus exceptional fibers is at most three. The only exception is that $Q \cong K^{2} \ltimes I$ has a Möbius strip base; but it also has its other fibration with Seifert data $\left\{D^{2},(2,1),(2,1)\right\}$.

We claim that we can recognize each possibility for $Q$ by a bounded number of applications of Theorem 8.5; the recognition algorithm is therefore in ER. Indeed, each case reduces to earlier cases when a putative essential surface is found. The most subtle case is case 5, where we can check whether a candidate torus in $Q$ is essential by checking that it is not compressible (case 1) and does not bound a thickened torus (case 3 ). Note that if $Q$ is a solid torus (case 1) or a thickened torus that is not glued to itself (case 3), or if $Q$ has an essential torus (case 5), then the collection $C$ in $W$ should be rejected.

In case 6 , we use Theorem 8.2 to determine if $W$ is small Seifert-fibered, and if so, its homeomorphism type.

If $Q$ is a thickened torus which is glued to itself, then $W$ is a torus bundle over a circle, and we can find its monodromy matrix $A \in \mathrm{SL}(2, \mathbb{Z})$ with a homology calculation. We can solve the conjugacy problem in $\operatorname{SL}(2, \mathbb{Z})$ using the usual trick that $\operatorname{PSl}(2, \mathbb{Z}) \cong C_{3} * C_{2}$, and thus determine the homeomorphism type of $W$. (Note that $W$ may be either Sol, Nil, or Euclidean.)

Otherwise we can piece together the JSJ decomposition $\{N\}$ of $W$ from the (often non-unique) atoroidal decomposition $\{Q\}$. In each remaining case $Q$ has either 0 Seifert fibrations (if it is hyperbolic), or 2 fibrations (if it is $K^{2} \ltimes I$ ), or 1 fibration (in all other Seifert-fibered cases). Using the recognition of $Q$, we can calculate its Seifert data and express the fibration of each boundary torus $T \subseteq \partial Q$ by the corresponding line $L \subseteq H_{1}(T)$. We can then piece together adjacent fibered components to make JSJ components, when the fibrations match. The Seifert data produced in this manner is not necessarily canonical, but canonicalizing it is straightforward.

\subsection{Algebraic algorithms}

We list several complexity bounds concerning algebraic numbers and solutions to algebraic equations.

Theorem 8.8 (Collins, Monk, Vorobiev-Grigoriev, Wüthrich [10, Thm. 4]). Suppose that a set $S \subseteq \mathbb{R}^{n}$ is defined by a finite set of polynomial equalities and inequalities over $\mathbb{Q}$. Then it is in ER to calculate a representative finite set $F \subseteq \widehat{\mathbb{Q}}^{n}$, with one point $p \in F$ in each connected component of $S$.

Theorem 8.8 is of course an ER version of Theorem 2.8. In the statement of the theorem, each element $\alpha \in \hat{\mathbb{Q}}$ described by its minimal polynomial $a(x) \in \mathbb{Z}[x]$ and an isolating interval $\alpha \in[b, c]$ that contains exactly one root of $a(x)$.

Lemma 8.9. If $\alpha \in \hat{\mathbb{Q}}$ is a non-zero complex root of a polynomial $a(x) \in \mathbb{Z}[x]$, then there is an ER upper bound on $|\alpha|$ and $\left|\alpha^{-1}\right|$. 
Proof. Let $n=\operatorname{deg} a$ and write

$$
a(x)=a_{n} x^{n}+a_{n-1} x^{n-1}+\cdots+a_{1} x+a_{0} .
$$

We can assume without loss of generality that $a_{0} \neq 0$. If $|\alpha|>\sum_{k} a_{k}$, then us that $\left|a_{n} \alpha^{n}\right|$ is larger than the total norm of all of the other terms, so by the triangle inequality, $a(\alpha) \neq 0$. This establishes $\sum_{k} a_{k}$ as an upper bound on $|\alpha|$. For the lower bound, we can observe that $\beta=\alpha^{-1}$ is a root of the polynomial

$$
b(x)=a_{0} x^{n}+a_{1} x^{n-1}+\cdots+a_{n-1} x+a_{n}=x^{n} a\left(x^{-1}\right) .
$$

We can thus repeat the argument.

If $x_{1}, \ldots, x_{n}$ are algebraic numbers, then we say that an algebraic number $z$ is an integral primitive element if each $x_{k}=f_{k}(z)$ for some integer polynomial $f_{k} \in \mathbb{Z}[x]$. It is a result of Galois that every finite set of algebraic numbers has a primitive element; we are interested in a computationally bounded version.

Theorem 8.10 (Koiran [25, Thm. 4]). If $x_{1}, \ldots, x_{n}$ are algebraic numbers, then they have an integer primitive element $z$ which can be computed in $\mathrm{ER}$, and such that polynomials $f_{k}$ with $f_{k}(z)=x_{k}$ can also be computed in $\mathrm{ER}$.

Koiran states the result in the form $x_{k}=f_{k}(z) / a_{k}$, where the denominator $a_{k}$ is an elementary recursive integer. However, it is not difficult to modify $z$ to eliminate these denominators. (Proof: Let $f \in \mathbb{Z}[x]$ be the minimal polynomial of $z$ and let

$$
z_{1}=\frac{z}{f(0) \prod_{k} a_{k}} .
$$

Then both $1 / a_{k}$ and $f_{k}(z)$ are expressible as integer polynomials in $z_{1}$. Therefore, so is their product.)

Lemma 8.11. If $h \in \mathbb{Z}[x]$ is an integer polynomial, then there is a prime $p$ that can be computed in ER such that $h(x)$ has a root in $\mathbb{Z} / p$.

Proof. If $d=\operatorname{deg} h$, then $h$ attains the values \pm 1 at most $2 d$ times. Therefore there is an integer $a$ with $|a| \leq d$ such that $h(a)$ is not \pm 1 , and we can let $p$ be a prime divisor of $h(a)$.

Using the results so far in this section, we obtain an elementary recursive version of Mal'cev's theorem, which says that finitely generated residually linear groups are residually finite. Our computational version requires a finitely present

Theorem 8.12. Let $\Gamma$ be a finitely presented group, let $g \in$ $\Gamma \backslash\{1\}$ be a non-trivial element given by a word $w$ in the generators of $\Gamma$, and suppose that there is a representation

$$
\rho: \Gamma \rightarrow \mathrm{GL}(n, \mathbb{C})
$$

that distinguishes $g$ from the identity. Then $\Gamma$ admits a finite representation

$$
\rho_{p}: \Gamma \rightarrow \mathrm{GL}(n, \mathbb{Z} / p)
$$

that distinguishes $g$ from the identity, where $p$ is a prime number. Moreover, we can find such a $p$ and $\rho_{p}$ in ER given the presentation of $\Gamma$, the word $w$, and the integer $n$.
Proof. A function from the generators of $\Gamma$ to $n \times n$ matrices forms a representation

$$
\rho: \Gamma \rightarrow \mathrm{GL}(n, \mathbb{C})
$$

if and only if the matrix entries satisfy equations that come from the relators of the presentation of $\Gamma$. We also want $\rho(g) \neq I$. To this end, we assume another matrix of variables $Y$ and impose the condition

$$
\operatorname{tr}(Y(\rho(g)-I))=1 .
$$

By hypothesis $\rho$ and $Y$ exist, and Theorem 8.8 then produces an algebraic, elementary recursive solution. By Theorem 8.10, the matrix entries are generated by an integer primitive element $z$, and by Lemma 8.11, we can replace $z$ by a residue $\alpha \in \mathbb{Z} / p$ for some prime $p$ computable in ER. We thus get a modular representation

$$
\rho_{p}: \Gamma \rightarrow \mathrm{GL}(n, \mathbb{Z} / p)
$$

and a matrix $Y_{p}$ over $\mathbb{Z} / p$ again with the same properties. Since $Y_{p}$ exists, $\rho_{p}(g)$ cannot be the identity.

Remark. Assuming the Generalized Riemann Hypothesis, Koiran's work implies Theorem 8.12 with a much better bound, namely that $\log (p)$ can be bounded by a polynomial in $n$, the length of the presentation of $\Gamma$, and the length of the word $w$.

\subsection{The small Seifert-fibered case}

In this section we will prove Theorem 8.2.

Let $N$ be a closed, oriented 3-manifold which has been recognized as irreducible and atoroidal by part of the algorithm in the proof of Theorem 8.1. We want to distinguish between the case that $N$ is small Seifert-fibered and the case that $N$ is hyperbolic; and in the former case, find its homeomorphism type. By Theorem 8.7, we also may as well assume that $N \neq \cong S^{3}$. We divide the proof into two cases, according to whether $\pi_{1}(N)$ is finite or infinite.

Proposition 8.13. It is in ER to determine if $\pi_{1}(N)$ is finite and compute its oriented homeomorphism type.

Proof. We first calculate whether $N$ is a lens space. We calculate $H_{1}(N)$ by applying the Smith normal form algorithm to its chain complex. If $H_{1}(N)$ is infinite, then $N$ is not small Seifert-fibered. Otherwise the cardinality of $H_{1}(N)$ is elementary recursive. We can calculate whether $N$ is a lens space by checking whether $H_{1}(N) \cong \mathbb{Z} / m$ is cyclic and calculating whether its abelian universal cover $\tilde{N}$ is isomorphic to $S^{3}$. To determine the parameter $n$ in the homeomorphism type of $N \cong L(m, n)$, we can calculate the Reidemeister torsion of the twisted homology of $N$ over the ring $\mathbb{Z}[\zeta]$, where $\zeta$ is an $m$ th root of unity. This is a determinant calculation which is a priori elementary recursive. Note that this torsion determines the oriented homeomorphism type of $N$.

If $N$ is spherical but not a lens space, then it has a finite covering space of order at most 12 which is a lens space. We 
thus obtain an elementary recursive bound on the cardinality of $\pi_{1}(N)$. Using a presentation of $\pi_{1}(N)$ obtained from the triangulation of $N$, we can search exhaustively among surjective homomorphisms $\phi: \pi_{1}(N) \rightarrow \Gamma$, where $\Gamma$ is a finite candidate for $\pi_{1}(N)$. For each such surjective homomorphism, we can build the corresponding covering space $\tilde{N}$ and calculate whether $\tilde{N} \cong S^{3}$. If this happens, then we know that $N \cong S^{3} / \Gamma$ as unoriented 3-manifolds.

Finally in the spherical case, we want to pass from the unoriented to the oriented homeomorphism type of $N$ when $N$ is spherical but not lens. (Note that every such $N$ is chiral.) As a first warm-up, recall that we can distinguish the lens space $L(4,1)$ from its reverse $L(4,-1)=L(4,3)$ by computing its Reidemeister torsion. As a second warm-up, we consider the simplest prism space $R(1,2)$ whose fundamental group $\Gamma=\pi_{1}(R(1,2))$ is the quaternionic 8-element group. We can build $R(1,2)$ as a coset space inside $\mathrm{SU}(2)$ :

$$
\Gamma \subseteq \mathrm{SU}(2) \quad R(1,2) \cong \mathrm{SU}(2) / \Gamma .
$$

The group $\Gamma$ has four cyclic subgroups of order 4 which are not conjugate in $\Gamma$ itself, but which are conjugate in $\mathrm{SU}(2)$. Matching this calculation to the Seifert data, $R(1,2)$ has three double covers which are all-oriented homeomorphic to $L(4,1)$. We can thus calculate the orientation of $N$ by calculating whether any double cover is $L(4,1)$ or $L(4,3)$.

In general if $N$ is spherical but not lens, then the quotient group $\pi_{1}(N) / Z\left(\pi_{1}(N)\right)$ is either a dihedral group, which is the prism case; or the isometry group of a Platonic solid: tetrahedral, octahedral, or icosahedral. In the case that $N \cong$ $R(m, n)$ and $m$ is odd, as well as in the Platonic cases, $\pi_{1}(N)$ has a unique subgroup isomorphic to the quaternionic group. Thus we can form the corresponding covering space $\tilde{N} \cong$ $R(1,2)$ and calculate its orientation as in the second warmup. Mean while if $N \cong R(m, n)$ and $m$ is even, then the center $Z\left(\pi_{1}(N)\right) \cong \mathbb{Z} /(2 m)$ has a unique subgroup of order 4 . Thus $\pi_{1}(N)$ has a canonically chosen cyclic subgroup of order 4 , and we can again form $\tilde{N}$ and calculate whether it is $L(4,1)$ or $L(4,3)$.

To prove Proposition 8.14, we will use a different combinatorial model of Seifert-fibered spaces than the one in Section 5.2.4. If $N$ is Seifert-fibered with base $F$, then we can consider a triangulation $\Theta$ of $F$ with the orbifold points placed at the vertices. For each triangle $\Delta \in \Theta$, we make a solid torus $\Delta \times S^{1}$ which we interpret as a chart for a circle bundle with structure group $S^{1}$. Then we can construct $N$ with an atlas of charts of this type. (It is an atlas with closed charts rather than open charts, but this is valid in context.) When two triangles $\Delta_{1}$ and $\Delta_{2}$ intersect in an edge, we glue the charts together with a transition map

$$
f_{12}: \Delta_{1} \cap \Delta_{2} \rightarrow S^{1} \cong \mathbb{R} / \mathbb{Z}
$$

We can assume that each transition map $f_{12}$ is affine-linear if lifted to $\mathbb{R}$, so that the value of $f_{12}$ lies in $\mathbb{Q} / \mathbb{Z}$, and its slop is also in $\mathbb{Q}$. Morevoer, it is not hard to convert the Seifert data for $N$ into these transition functions, for any triangulation of $F$. Finally, note that if $p \in F$ is an orbifold point of order $a$

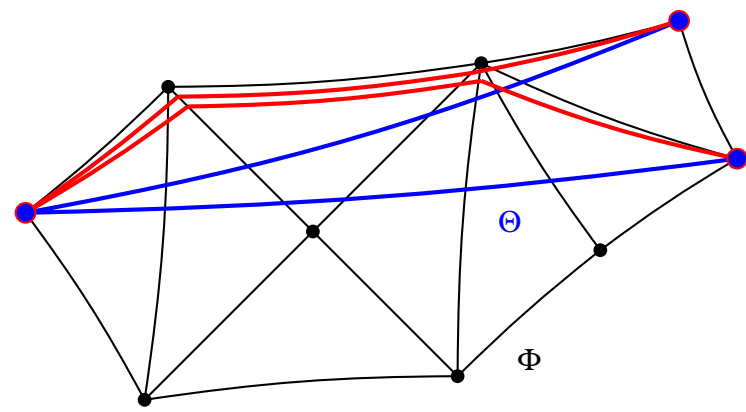

Figure 3. Isotopy of a triangulation $\Theta$ of $\tilde{F}$ (initially in blue) so that in its new position (in red), its edges are parallel to a $\pi_{1}(N)$-invariant triangulation $\Phi$.

and $\Delta \in \Theta$ is any triangle that has $p$ as a vertex, then the gluing maps between charts glue the circle over $p$ in such a way that it shortens by a factor of $a$ and becomes a singular fiber of $N$.

Proposition 8.14. It is in ER to determine if $N$ is small Seifertfibered with infinite $\pi_{1}(N)$, and if so, compute its oriented homeomorphism type.

Proof. If $\pi_{1}(N)$ is infinite and $N$ is small Seifert-fibered, then the base $F$ of $N$ is a 2-sphere with orbifold points of order $a_{1} \geq a_{2} \geq a_{3}$ with

$$
\frac{1}{a_{1}}+\frac{1}{a_{2}}+\frac{1}{a_{3}} \leq 1
$$

In the equality case $F$ is Euclidean and $N$ is either Euclidean or Nil; otherwise $F$ is hyperbolic and the geometry of $N$ is either $\mathbb{H}^{2} \times \mathbb{R}$ or Isom $\left(\mathbb{H}^{2}\right)$. The orbifold fundamental group $\pi_{1}(F)$ is a von Dyck group $D\left(a_{1}, a_{2}, a_{3}\right)$ which is the orientationpreserving subgroup of index two in the corresponding triangle group $\Delta\left(a_{1}, a_{2}, a_{3}\right)$ in either $\operatorname{Isom}\left(\mathbb{E}^{2}\right)$ or $\operatorname{Isom}\left(\mathbb{H}^{2}\right)$, and $\pi_{1}(N)$ is a central extension of $D\left(a_{1}, a_{2}, a_{3}\right)$ by $\mathbb{Z}$.

We first consider the case in which $F$ is Euclidean, which implies that $\left(a_{1}, a_{2}, a_{3}\right)$ is either $(3,3,3),(4,4,2)$, or $(6,3,2)$. In this case there is a homomorphism

$$
\phi: \pi_{1}(N) \rightarrow G
$$

where the target group is respectively the dihedral group $D_{3}$, $D_{4}$, or $D_{6}$, such that the corresponding regular cover $\tilde{N}$ is a circle bundle over a torus. Given a putative choice for $G$ and $\phi$, we can construct the regular cover $\tilde{N}$ and apply the large Seifert-fibered case of Theorem 8.1 to recognize it. If $\tilde{N}$ is indeed a circle bundle over a torus, then we know that $N$ must be small Seifert-fibered with a Euclidean base, and the remaining question is to confirm that $G$ and $\phi$ were correctly chosen and thus compute the specific Seifert data of $N$.

Either $\tilde{N}$ is $S^{1} \times S^{1} \times S^{1}$ (so that $N$ itself is Euclidean), or it is a circle bundle over $S^{1} \times S^{1}$ with a non-trivial Euler number and thus has Nil geometry. We thus obtain an explicit form of $\pi_{1}(\tilde{N})$ which is either $\mathbb{Z}^{3}$ or a central extension of $\mathbb{Z}^{2}$ by $\mathbb{Z}$. At the same time, since the recognition of the structure of $\tilde{N}$ is based on normal surface theory, it thus yields a retriangulation of $\tilde{N}$ in ER from the one triangulation induced by the input triangulation of $N$ to one that reveals the Seifert structure. 
Therefore we obtain an explicit (and elementary recursive) description of $\pi_{1}(N)$ as an extension of the finite group $G$ by $\pi_{1}(N)$. We can thus match $\pi_{1}(N)$ to the corresponding model Seifert-fibered space to determine the unoriented homeomorphism type of $N$. Finally we have to calculate the oriented homeomorphism type. In the Euclidean case, the recognition of $\tilde{N}$ gives us an orientation of $\pi_{1}(\tilde{N}) \cong \mathbb{Z}^{3}$. Similarly in the Nil case, when $\pi_{1}(\tilde{N})$ is an extension of $\mathbb{Z}^{2}$ by $\mathbb{Z}$, the orientation of $\tilde{N}$ still gives an orientation of both the center $\mathbb{Z}$ and the quotient $\mathbb{Z}^{2}$, up to switching both orientations. This fixes an orientation of $N$ itself.

The argument when the base $F$ is hyperbolic is similar to the Euclidean case but more complicated. In this case, $\pi_{1}(N)$ has a non-trivial homomorphism to $\operatorname{Isom}\left(\mathbb{H}^{2}\right)$, which in turn embeds in $\operatorname{SL}(2, \mathbb{C})$, so Theorem 8.12 tells us that $\pi_{1}(N)$ has a non-trivial finite quotient $G$ which we can find in ER even if we do not know the linear representation that explains that it exists.

We construct the finite cover $\tilde{N}$ of $N$ corresponding to the quotient map $\phi: \pi_{1}(N) \rightarrow G$, and we apply part of Theorem 8.1 to determine if $\tilde{N}$ is large Seifert-fibered, and if so calculate its fibration and its base $\tilde{F}$. As before, we can first learn from this whether $N$ is indeed small Seifert-fibered. Second, as before this part of Theorem 8.1 gives us an ER retriangulation from the initial triangulation of $\tilde{N}$ as a covering space of $N$, to a triangulation that reflects its Seifert fibration. In particular, we obtain a triangulation $\Theta$ of $\tilde{F}$ together with an atlas of charts to describe $\tilde{N}$. Third, we can choose an orientation of $\tilde{F}$ and an orientation of the circle fibers so that the two orientations together are consistent with the orientation of $\tilde{N}$ inherited from $N$. Fourth, using the orbifold point orders of $\tilde{F}$ and the cardinality of $G$, we obtain an ER upper bound on $a_{1}, a_{2}$, and $a_{3}$.

For each candidate for $\left(a_{1}, a_{2}, a_{3}\right)$, the given representation of $D\left(a_{1}, a_{2}, a_{3}\right)$ in $\operatorname{Isom}\left(\mathbb{H}^{3}\right)$ is rigid. It is still rigid even as a representation of $\pi_{1}(N)$, because any nearby representation must still annihilate the kernel $Z\left(\pi_{1}(N)\right)$. Passing to the covering space $\tilde{F}$, we obtain a preferred hyperbolic structure on $\tilde{F}$ and we can realize $\Theta$ as a geometric triangulation. (In two dimensions, every triangulation of a hyperbolic surface is geometric.) Now Theorem 8.8, combined with the fact that the retriangulation of $\tilde{N}$ is in ER, gives us an ER upper bound on the lengths of the edges of $\Theta$. At the same time, we get a second (possibly generalized) triangulation $\Phi$ of $\tilde{F}$ by tiling it by lifts of the triangular fundamental domain of the triangle group $\Delta\left(a_{1}, a_{2}, a_{3}\right)$; this triangulation is both geometric and $\pi_{1}(N)$-invariant. As illustrated in Figure 3, we can isotop $\Theta$ so that its edges run parallel to $\Phi$. (Since this isotopy cannot introduce crossings, and since two edges of $\Theta$ might follow some of the same edges of $\Phi$ as illustrated, they cannot land exactly onto these edges.) This isotopy shows that $\Phi$ and $\Theta$ have a mutual refinement that can be found in ER. Thus we can search over retriangulations of $\tilde{F}$ in ER until we find one that is $\pi_{1}(N)$-invariant. We can then use this to compute the Seifert structure on $N$, moreover preserving the orientation information inherited from the fibration of $\tilde{N}$.

\subsection{The hyperbolic case}

To prove Theorem 8.3, we will need a quick mutual corollary of Theorem 8.8 and the proof of Proposition 3.5.

Corollary 8.15. If $\Theta_{1}$ is a finite simplicial complex with $n_{1}$ simplices (of arbitrary dimension) and $n_{2} \geq n_{1}$, then it is in ER to produce a complete list of geometric subdivisions $\Theta_{2}$ of $\Theta_{1}$ with $n_{2}$ simplices.

Proof of Theorem 8.3. Let $\Theta$ be the input triangulation of $N$ as a compact manifold, and let $\Theta^{*}$ be the result of adding a cone to each component of $\partial N$ to make a semi-ideal combinatorial triangulation of $N^{*}$. The manifold $N^{*}$ also has a hyperbolic structure which we interpret as a separate manifold. We rename the hyperbolic version $X$ and assume a homeomorphism

$$
f: N^{*} \rightarrow X \text {. }
$$
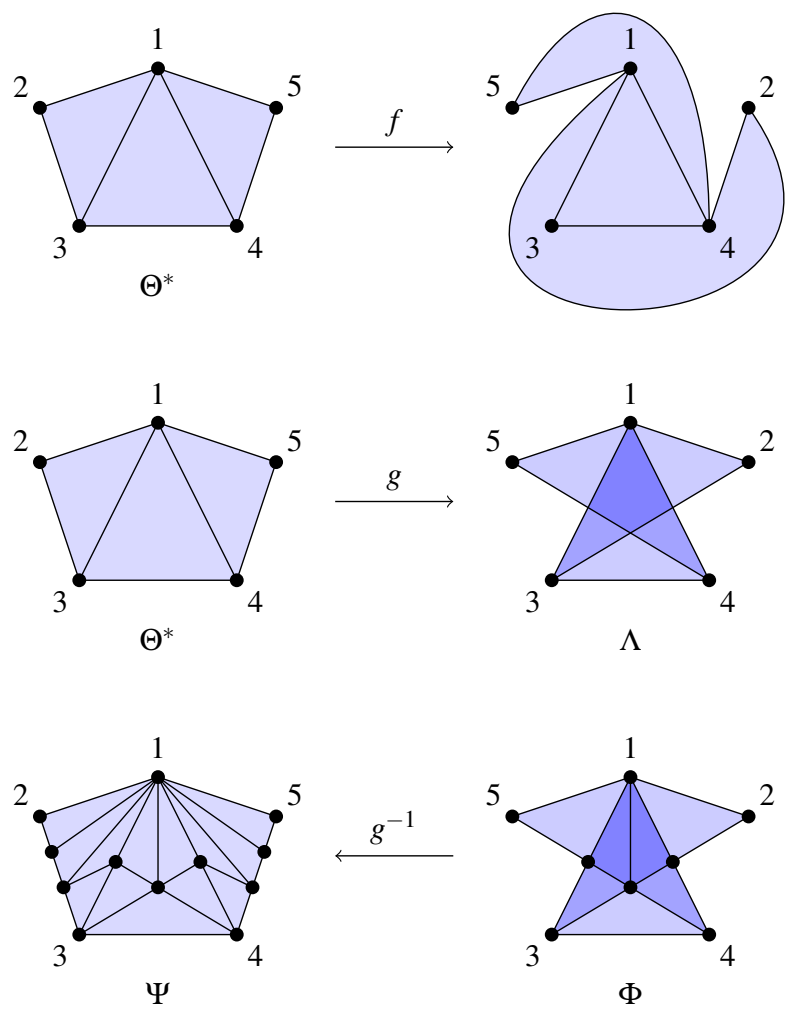

Figure 4. We straighten $f$ to $g$, then subdivide the image, and finally subdivide the domain to make $g$ a simplicial map. In the proof the image is barycentrically subdivided, but any refinement which is a triangulation suffices.

We fix the vertices of $N^{*}$ in the map $f$, and straighten all of the tetrahedra, to make a map $g$ that represents $\Theta^{*}$ as a self-intersecting geometric triangulation of $X$. Since $g$ is homotopic to $f$ (or properly homotopic of $N^{*}$ is not compact), it has (proper) degree 1. The simplices of $g$ may be flat or flipped over, but $g$ has degree 1 since it is homotopic to $f$. The self-intersections of $g\left(\Theta^{*}\right)$ yield a cellulation $\Lambda$ of $X$ with convex cells. Thus $\Lambda$ has a barycentric subdivision $\Phi$ which 
is a geometric triangulation of $X$. Also let $\Psi=g^{-1}(\Phi)$. Then $\Psi$ is a refinement of the triangulation $\Theta^{*}$, and $g$ is now a simplicial map from $\Psi$ to $\Phi$. See Figure 4. (The figure uses a simplicial refinement of the self-intersections which is simpler than barycentric subdivision; this is not important for the proof.)

Now suppose that we do not know the hyperbolic structure of $N$, only that it must have one because it prime, atoroidal, and acylindrical. If we are given $\Psi$ as a combinatorial refinement of the triangulation $\Theta^{*}$, then we can search for $\Phi$ as a simplicial quotient of $\Psi$, such that we can solve the hyperbolic gluing equations for $\Phi$ to recognize it as a geometric triangulation of a hyperbolic manifold $X$. We obtain a candidate map $g: N^{*} \rightarrow X$. If $g$ has degree 1 , and there is also a degree 1 map $h: X \rightarrow N^{*}$, then Mostow rigidity tells us that $g$ and $h$ are both homotopy equivalences and that $X$ and $N^{*}$ are homeomorphic. (Note that there can be a degree 1 map in one direction between two hyperbolic 3-manifolds that is not a homotopy equivalence [5], even though this cannot happen in the case of hyperbolic surfaces.) We can search for $h$ by the same method of simplicial subdivision that we used to find g. This establishes an algorithm to calculate the hyperbolic structure of $N^{*}$.

We claim that a modified version of this algorithm is in ER. We first consider ER candidates for the map $g$. To do this, we make a non-commutative cocycle $\alpha \in C^{1}(N ; G)$ as in the proof of Corollary 5.10, where

$$
G=\operatorname{Isom}^{+}\left(\mathbb{H}^{3}\right) \cong \operatorname{ISO}(3,1),
$$

and with the extra restriction that $\alpha$ is parabolic on each component of $\partial N$. These cocycle equations are algebraic, so Theorem 8.8 guarantees a representative set of solutions. By Mostow or Calabi-Weil rigidity, one of components of the solution space yields a discrete homomorphism

$$
\rho: \pi_{1}(N) \rightarrow \operatorname{Isom}^{+}\left(\mathbb{H}^{3}\right)
$$

that describes the hyperbolic geometry of $X$. If we assign some point $p \in \mathbb{H}^{3}$ to one of the vertices of $\Theta$, then in the closed case, its orbit under $\alpha$ is in ER and can be extended on each simplex of $\Theta$ to the map $g$. In the cusped case, there are also ideal vertices whose position on the sphere at infinity can be calculated from $\alpha$ as well.

If $N$ has boundary, then we also want a truncated version of $\Theta^{*}$ which is larger than the original $\Theta$, and slightly different from the horospheric truncation description in Section 7. If that $\Delta \in \Theta^{*}$ is semi-ideal, then let $p$ be its ideal vertex, let $F$ be the hyperplane containing the face of $\Delta$ opposite to $p$, and let $F^{\prime}$ be the hypersphere at distance $\log (2)$ from $F$ which is on the same side as $p$. Then we truncate $\Delta$ with $F^{\prime}$ to make $\Delta^{\prime}$; or if $\Delta \in \Theta^{*}$ is a non-ideal tetrahedron, we let $\Delta^{\prime}=\Delta$. We let $X^{\prime} \subseteq X$ be the union of all $\Delta^{\prime}$. (In the closed case, we obtain $X^{\prime}=X$.) $X^{\prime}$ can have a complicated shape because the truncations are usually mismatched, but we can calculate the positions of its vertices, and it is easy to confirm that it has at least half of the volume of $X$.

Our algorithm does not know which cocycle $\alpha$ gives us a desired $g$ and we do not compute this directly. Instead, we can calculate an ER bound for its data complexity, using the complexity bounds in the statement of Theorem 8.8.

In particular, the existence of the map $g$ gives us ER bounds on the parameters used in the third proof of Lemma 7.1. Using Lemma 8.9, the existence of $g$ yields an ER upper bound on the diameter $\ell$ of $X^{\prime}$ and then a lower bound on its injectivity radius $r$.

We can now follow the first proof of Theorem 6.1. If $\Delta_{1}, \Delta_{2} \in \Theta^{*}$ are two tetrahedra, then the intersection complexity of $g\left(\Delta_{1}\right)$ and $g\left(\Delta_{2}\right)$ is no worse than that of $g\left(\Delta_{1}^{\prime}\right)$ and $g\left(\Delta_{2}^{\prime}\right)$, and is bounded by an ER function of $\ell$ and $r$. This yields an ER bound on the complexity of the refinements $\Phi$ and $\Psi$. Recall that $\Psi$ is a refinement of $\Theta^{*}$, which is a slightly modified version of the input description of $N$. Having bounded the complexity of $\Psi$, we can search for it using Corollary 8.15 and solve for $\Phi$ and its geometry. We can also discard $g$ if it does not have degree 1 .

Thus far, the algorithm finds an ER collection of candidate maps $g: X^{*} \rightarrow N$ of degree 1 , where $N$ varies as well as $g$. At least one of these maps is a homotopy equivalence. Instead of finding an inverse $h$, We can repeat the algorithm to look for degree one maps among the target manifolds $\{N\}$. This induces a transitive relation among these manifolds. If $N$ is chosen at the top of this relation, then the associated map $g$ : $X^{*} \rightarrow N$ must be a homotopy equivalence.

To solve the isomorphism problem, we find geometric triangulations $\Phi_{1}$ and $\Phi_{2}$ of the manifolds $N_{1}^{*}$ and $N_{2}^{*}$. We can again follow the first proof of Theorem 6.1, except now with an ER bound on the complexity of $\Phi_{1}$ and $\Phi_{2}$, and we can again use Corollary 8.15. The same argument applies for the calculation of the isometry group of a single $N^{*}$.

\section{OPEN PROBLEMS}

Theorem 1.2, together with the fact that ER is a fairly generous complexity class, suggests the following conjectures.

Conjecture 9.1. If $M$ is a closed, Riemannian 3-manifold, then Ricci flow with surgery on $M$ can be accurately simulated in $\mathrm{ER}$.

In other words, we conjecture that Perelman's proof of geometrization can be placed in ER.

Conjecture 9.2. Every closed, hyperbolic manifold $N$ has a finite-sheeted Haken covering which is computable in ER.

In other words, we conjecture that the statement of the virtual Haken conjecture, now the theorem of Agol et al [1], can be placed in ER. Maybe the known proof can be as well.

Conjecture 9.3. Any two triangulations of a closed 3manifold $M$ have a mutual refinement computable in $\mathrm{ER}$.

Conjecture 9.3 does not follow from our proof of Theorem 1.2, because the algorithm in Theorem 8.3 only establishes a simplicial homotopy equivalence and then relies on Mostow rigidity. However, the rest of the proof of Theorem 1.2 uses a bounded number of normal surface dissections, 
which does establish an ER mutual refinement according to the arguments of Mijatović [31, 32]. Also, Conjecture 9.2 and the Haken case of Conjecture 9.3 would together imply the hyperbolic case of Conjecture 9.3, which would then imply the full conjecture. Mijatović [33] also established that any two triangulations of a fiber-free Haken 3-manifold have a primitive recursive mutual refinement.

Cases 3 and 4 of Proposition 2.6 are expected to be false for typical bounds on complexity that are better than ER. Thus, in discussing further improvements to Theorem 1.2, we should consider qualitative complexity classes, such as the famous NP, rather than just bounds on execution time. For one thing,
ER is the union of an alternating, nested sequence of time and space complexity classes, as follows:

$$
\mathrm{P} \subseteq \mathrm{PSPACE} \subseteq \mathrm{EXP} \subseteq \mathrm{EXPSPACE} \subseteq \mathrm{EEXP} \subseteq \cdots
$$

Here $\mathrm{P}$ is the set of decision problems that can be solved in deterministic polynomial time; PSPACE is solvability in polynomial space with unrestricted (but deterministic) computation time; EXP is deterministic time $\exp (\operatorname{poly}(n))$; etc. The author does not know where a careful version of our proof of Theorem 1.2 would land in this hierarchy.
[1] Ian Agol, The virtual Haken conjecture, Doc. Math. 18 (2013), 1045-1087, arXiv:1204.2810, With an appendix by Ian Agol, Daniel Groves, and Jason Manning. 9

[2] Ian Agol, Joel Hass, and William Thurston, The computational complexity of knot genus and spanning area, Trans. Amer. Math. Soc. 358 (2006), no. 9, 3821-3850, arXiv:math/0205057. 8.2

[3] Sanjeev Arora and Boaz Barak, Computational complexity: a modern approach, Cambridge University Press, Cambridge, 2009. 2, 2.2

[4] Matthias Aschenbrenner, Stefan Friedl, and Henry Wilton, Decision problems for 3-manifolds and their fundamental groups, Geom. Topol. Monogr. 19 (2015), 201-236, arXiv:1405.6274. $1,1.1$

[5] Michel Boileau and Shicheng Wang, Non-zero degree maps and surface bundles over $S^{1}$, J. Differential Geom. 43 (1996), no. 4, 789-806. 8.5

[6] Marc Culler and Nathan Dunfield, SnapPy, http://www.math.uic.edu/t3m/SnapPy/. 1.1

[7] Robert D. Edwards, Suspensions of homology spheres, 1975, arXiv:math/061057. 3

[8] David B. A. Epstein, James W. Cannon, Derek F. Holt, Silvio V. F. Levy, Michael S. Paterson, and William P. Thurston, Word processing in groups, Jones and Bartlett Publishers, 1992. 1, 1.1

[9] David Gabai, Robert Meyerhoff, and Peter Milley, Volumes of hyperbolic 3-manifolds, The Poincaré conjecture, Clay Math. Proc., vol. 19, American Mathematical Society, 2014, arXiv:0705.4325, pp. 65-79. 7

[10] Dima Yu. Grigoriev, Computational complexity in polynomial algebra, Proceedings of the International Congress of Mathematicians, Vol. 1, 2 (Berkeley, Calif., 1986), Amer. Math. Soc., 1987, pp. 1452-1460. 1, 8.1, 8.8

[11] Mikhael Gromov, Hyperbolic groups, Essays in group theory, Math. Sci. Res. Inst. Publ., vol. 8, Springer, 1987, pp. 75-263. 1

[12] Wolfgang Haken, Ein Verfahren zur Aufspaltung einer 3Mannigfaltigkeit in irreduzible 3-Mannigfaltigkeiten, Math. Z. 76 (1961), 427-467.

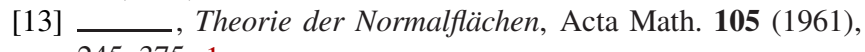
245-375. 1

[14] Joel Hass and Greg Kuperberg, A mixed NP certificate to show that a 3-manifold is not a 3-sphere, in preparation. 8.6

[15] Ivan Izmestiev, Infinitesimal rigidity of convex polyhedra through the second derivative of the Hilbert-Einstein functional, Canad. J. Math. 66 (2014), no. 4, 783-825,
arXiv:1105.5066. 5.10

[16] William Jaco, Lectures on three-manifold topology, CBMS Regional Conference Series in Mathematics, vol. 43, American Mathematical Society, 1980. 5.12, 6.3

[17] William Jaco, David Letscher, and J. Hyam Rubinstein, Algorithms for essential surfaces in 3-manifolds, Topology and geometry: commemorating SISTAG, Contemp. Math., vol. 314, Amer. Math. Soc., 2002, pp. 107-124. 8.1, 8.1, 8.2

[18] William Jaco and Jeffrey L. Tollefson, Algorithms for the complete decomposition of a closed 3-manifold, Illinois J. Math. 39 (1995), no. 3, 358-406. 1, 1., 2., 3., 4.

[19] William H. Jaco and Peter B. Shalen, Seifert-fibered spaces in 3-manifolds, Mem. Amer. Math. Soc. 21 (1979), no. 220, viii+192. 5.2

[20] Klaus Johannson, Homotopy equivalences of 3-manifolds with boundaries, Lecture Notes in Mathematics, vol. 761, Springer, 1979. 5.2

[21] Leonid V. Kantorovich, Functional analysis and applied mathematics, Vestnik Leningrad. Univ. 3 (1948), no. 6, 3-18. 5.3

[22] Michael Kapovich, Hyperbolic manifolds and discrete groups, Progress in Mathematics, vol. 183, Birkhäuser, 2001. 5.9

[23] R. C. Kirby and L. C. Siebenmann, On the triangulation of manifolds and the Hauptvermutung, Bull. Amer. Math. Soc. 75 (1969), 742-749. 3

[24] H. Kneser, Geschlossene Flächen in dreidimensionalen Mannigfaltigkeiten, Jahresbericht Math. Verein. 28 (1929), 248260. 1, 5.1

[25] Pascal Koiran, Hilbert's Nullstellensatz is in the polynomial hierarchy, J. Complexity 12 (1996), no. 4, 273-286, DIMACS TR 96-27, Special issue for FOCM 1997. 8.10

[26] Dmitry Kozlov, Combinatorial algebraic topology, Algorithms and Computation in Mathematics, vol. 21, Springer, Berlin, 2008. 5.5

[27] Tao Li, An algorithm to find vertical tori in small Seifert fiber spaces, Comment. Math. Helv. 81 (2006), no. 4, 727-753, arXiv:math/0209107. 8.1

[28] W. B. R. Lickorish, Simplicial moves on complexes and manifolds, Proceedings of the Kirbyfest (Berkeley, CA, 1998), Geom. Topol. Publ., Coventry, 1999, arXiv:math/9911256, pp. 299-320. 3

[29] Jason Manning, Algorithmic detection and description of hyperbolic structures on closed 3-manifolds with solvable word problem, Geom. Topol. 6 (2002), 1-25, arXiv:math/0102154. $1,1.1,1.1,5.3$

[30] Robert Meyerhoff, A lower bound for the volume of hyperbolic 3-manifolds, Canad. J. Math. 39 (1987), no. 5, 1038-1056. 7 
[31] Aleksandar Mijatović, Simplifying triangulations of $S^{3}$, Pacific J. Math. 208 (2003), no. 2, 291-324, arXiv:math/0008107. 1, 9

[32] - Triangulations of Seifert fibred manifolds, Math. Ann. 330 (2004), no. 2, 235-273, arXiv:math/0301246. 1, 9

[33] - Triangulations of fibre-free Haken 3-manifolds, $\mathrm{Pa}-$ cific J. Math. 219 (2005), no. 1, 139-186, arXiv:math/0306116. 9

[34] John Milnor, A unique decomposition theorem for 3-manifolds, Amer. J. Math. 84 (1962), 1-7. 5.1

[35] John W. Morgan and Frederick Tsz-Ho Fong, Ricci flow and geometrization of 3-manifolds, University Lecture Series, vol. 53, American Mathematical Society, 2010. 5.1

[36] Alexander Nabutovsky and Shmuel Weinberger, Algorithmic aspects of homeomorphism problems, Tel Aviv Topology Conference: Rothenberg Festschrift (1998), Contemp. Math., vol. 231, Amer. Math. Soc., 1999, arXiv:math/9707232, pp. 245250. 3

[37] John W. Neuberger, The continuous Newton's method, inverse functions, and Nash-Moser, Amer. Math. Monthly 114 (2007), no. $5,432-437.5 .3,5.8$

[38] Walter D. Neumann and Don Zagier, Volumes of hyperbolic three-manifolds, Topology 24 (1985), no. 3, 307-332. 5.3

[39] Bjorn Poonen, Undecidable problems: a sampler, ch. 10, pp. 211-241, Cambridge University Press, 2014, arXiv:1204.0299. 3

[40] J. Hyam Rubinstein, An algorithm to recognize the 3-sphere, Proceedings of the International Congress of Mathematicians, Vol. 1, 2 (Zürich, 1994), Birkhäuser, 1995, pp. 601-611. 3, 8.7

[41] Sage open-source mathematical software system, http://www . sagemath.org/. 2.3

[42] Saul Schleimer, Sphere recognition lies in NP, Lowdimensional and symplectic topology, Proc. Sympos. Pure Math., vol. 82, Amer. Math. Soc., 2011, arXiv:math/0407047, pp. 183-213. 8.2

[43] Peter Scott and Hamish Short, The homeomorphism problem for closed 3-manifolds, Algebr. Geom. Topol. 14 (2014), no. 4, 2431-2444, arXiv:1211.0264. 1, 1.1, 7

[44] Abraham Seidenberg, A new decision method for elementary algebra, Ann. of Math. (2) 60 (1954), 365-374. 2.8

[45] Herbert Seifert, Topology of 3-dimensional fibered spaces, in Seifert and Threlfall: a textbook of topology, Academic Press, 1980, pp. 359-422. 4

[46] Zlil Sela, The isomorphism problem for hyperbolic groups. I, Ann. of Math. (2) 141 (1995), no. 2, 217-283. 1, 1.1

[47] Alfred Tarski, A decision method for elementary algebra and geometry, Tech. Report R-109, The Rand Corporation, 1948. 2.8

[48] Abigail Thompson, Thin position and the recognition problem for $S^{3}$, Math. Res. Lett. 1 (1994), no. 5, 613-630. 3, 8.7

[49] William P. Thurston, Three-dimensional manifolds, Kleinian groups and hyperbolic geometry, Bull. Amer. Math. Soc. (N.S.) 6 (1982), 357-381. 1.1, 1, 1.1, 5.1

[50] - On proof and progress in mathematics, Bull. Amer. Math. Soc. (N.S.) 30 (1994), no. 2, 161-177, arXiv:math/9404236. 1

[51] Friedhelm Waldhausen, Gruppen mit Zentrum und 3dimensionale Mannigfaltigkeiten, Topology 6 (1967), 505-517. $1.1,6.3$

[52] _ Recent results on sufficiently large 3-manifolds, Algebraic and geometric topology (Proc. Sympos. Pure Math., Stanford Univ., Stanford, Calif., 1976), Part 2, Proc. Sympos. Pure Math., XXXII, Amer. Math. Soc., 1978, pp. 21-38. 1.1

[53] Jeffrey Weeks, SnapPea, a hyperbolic structures computation program, http://www.geometrygames.org/SnapPea/. 1.1
[54] The Complexity Zoo, http://www.complexityzoo.com/. 2 